\title{
Large-scale all-optical dissection of motor cortex connectivity reveals a segregated functional organization of mouse forelimb representations
}

\author{
Francesco Resta ${ }^{1,2, \#, *}$, Elena Montagni ${ }^{1,2, \#, ~ G i u s e p p e ~ d e ~ V i t o ~}{ }^{1,3}$, Alessandro \\ Scaglione $^{1,2}$, Anna Letizia Allegra Mascaro ${ }^{1,4, *}$, Francesco Saverio Pavone ${ }^{1,2,5}$ \\ 1. European Laboratory for Non-Linear Spectroscopy, University of Florence, Sesto Fiorentino \\ 50019, Italy \\ 2. Department of Physics and Astronomy, University of Florence, Sesto Fiorentino 50019, Italy \\ 3. Department of Neuroscience, Psychology, Pharmacology and Child Health (NEUROFARBA), \\ University of Florence, Florence 50139, Italy \\ 4. Neuroscience Institute, National Research Council, Pisa 56124, Italy \\ 5. National Institute of Optics, National Research Council, Sesto Fiorentino 50019, Italy \\ \# = equally contributed \\ * $=$ corresponding authors
}

\section{ABSTRACT}

In rodent motor cortex, the rostral forelimb area (RFA) and the caudal forelimb area (CFA) are major actors in orchestrating the control of forelimb complex movements. However, their intrinsic connections and reciprocal functional organization are still unclear, limiting our understanding of how the brain coordinates and executes voluntary movements. Here we causally probed cortical connectivity and activation patterns triggered by transcranial optogenetic stimulation of ethologically relevant complex movements exploiting a novel largescale all-optical method in awake mice. Results show specific activation features for each movement class, providing evidence for a segregated functional organization of CFA and RFA. Importantly, we identified a second discrete lateral grasping representation area, namely lateral forelimb area (LFA), with unique connectivity and activation patterns. Therefore, we propose the LFA as a distinct motor representation in the forelimb somatotopic motor map. 


\section{INTRODUCTION}

Complex motor behaviors are a combination of discrete and rhythmic movements ${ }^{1-3}$. Discrete movements, such as reaching and grasping, are described as continuous movements from a starting to an ending point in space, while rhythmic movements are periodic, repetitive, and stereotyped, like locomotion or licking ${ }^{1,4}$. In rodents, forelimb movements are controlled by two distinct cortical functional areas: the caudal forelimb area (CFA) and the rostral forelimb area (RFA). Although a large number of works elucidated the anatomical structure and functional outputs of these subregions, their intrinsic connections and reciprocal functional role are unclear ${ }^{5}$.

The functional organization of the motor cortex is canonically investigated combining cortical electrical stimulation and behavioral readout to map cortical movement representations ${ }^{6-11}$. However, the electrophysiological approach is invasive, limited in spatial resolution and lacks cellular population selectivity, thus it is being progressively replaced by optogenetic, which overcomes these limitations ${ }^{4,12-14}$. Light-based motor maps (LBMMs) are powerful tools to study motor cortex topography, however important questions remain concerning the mechanisms that coordinate the activity of different functional areas during voluntary movement execution. In particular, the relative contribution and hierarchic relationship of the RFA and CFA in control forelimb movements are still debated. To answer these questions, it would be beneficial to combine motor mapping with large-scale monitoring of cortical activity. Wide-field fluorescence imaging of voltage-sensitive dyes (VSDs) or genetically-encoded calcium indicators (GECls) represents the most effective strategy to study motor cortex activity at mesoscale level ${ }^{15-18}$. Although VSDs present high temporal resolution (sub-milliseconds), the use of dyes rules out the possibility to target specific cell populations and to perform longitudinal studies, due to the invasive nature of the dye application. Despite the slower kinetics, GECls overcome these limitations and show a higher signal-to-noise ratio, resulting in the best choice to study cortical connectivity at the mesoscale level in awake mice. Nevertheless, a crucial factor to consider when combining fluorescence imaging and optogenetics in all-optical configurations is the excitation/absorption spectral overlap that leads to crosstalk between imaging and photostimulation ${ }^{19}$.

Here we first established a crosstalk-free large-scale all-optical experimental configuration combining wide-field fluorescence imaging of the red-shifted GECI jRCaMP1a and optogenetic stimulation of Channelrhodopsin-2 (ChR2) to perform light-based motor mapping of complex movements in awake mice while monitoring the cortical activity of excitatory neurons. Thanks to this low-invasive transcranial method, we investigated RFA and CFA effective connectivity and functional dependencies by causally dissecting the cortical activity patterns triggered by optogenetic stimulation. Our results show a modular organization of the movement-specific activated areas and peculiar activity propagation hallmarks during forelimb complex movement execution. Importantly, we identified a discrete lateral and caudal grasping cortical representation expressing distinct topographic and connectivity features. 


\section{RESULTS}

To explore the intrinsic connections and reciprocal functional role of RFA and CFA, we implemented a novel large-scale all-optical method to causally dissect the cortical activity patterns triggered by optogenetic stimulation of two ethologically relevant forelimb movements. Initially, we mapped the cortical representation of two distinct complex movements, a grasping-like movement and a locomotion-like movement optogenetically evoked in RFA and CFA respectively. Next, we recorded the mesoscale cortical response during movement execution. Finally, we quantified the activation maps related to the movements somatotopy to study the RFA and CFA connectivity.

\section{Wide and long-term stable cortical transfection of both jRCaMP1a and ChR2 in mouse motor cortex}

In mice expressing optogenetic actuators in the motor cortex, several classes of limb movements can be evoked depending on the stimulated site ${ }^{4,12,13}$. To investigate large-scale cortical activity underlying the optogenetically-evoked movements, we infected the frontal right cortical hemisphere of C57/B6 mice with adeno-associated viruses (AAV) carrying the redshifted GECI jRCaMP1a and the optogenetic actuator ChR2. The optical setup consisted of a double-path illumination system integrated into a custom-made wide-field fluorescence microscope for parallel laser stimulation and wide-field cortical imaging (fig. 1a). To evaluate the transfection extension and its long-term stability, the spatial fluorescence intensity profiles were calculated (fig. 1b). In line with our previous observations ${ }^{20}$, we found that ChR2 and jRCaMP1a expression covered all the motor cortices, and their expression levels were highly stable over several weeks (fig. 1b; supplementary fig. 3). Moreover, the expression profiles on histological brain slices showed that the transfection of jRCaMP1a and ChR2 were restricted to the motor cortex reaching all the cortical layers (fig. 1d). Besides, to evaluate the synapsintargeted jRCaMP1a transfection efficiency, brain slices were stained with the neuronal marker NeuN, revealing that the jRCaMP1a+ neurons were $70.2 \pm 4.9 \%$ of the $\mathrm{NeuN}^{+}$cells (fig. $1 \mathrm{e}$ ). Therefore, our injection method leads to a wide transfection that covers all the motor cortices and is sufficiently stable to perform experiments several weeks after injection with stable expression levels.

\section{Wide-field imaging of jRCaMP1a does not induce ChR2 cross-activation}

The all-optical approach we chose to visualize cortical activation during optogeneticallyevoked complex movements combines blue-activated opsins and red-shifted GECls ${ }^{21-25}$. To date, there are two main red-shifted GECl families, comprising the RGECO and RCaMP variants that are based on mApple and mRuby protein respectively. Despite RGECOs show higher $\mathrm{Ca}^{2+}$ affinity and larger dynamic range compared to RCaMPs, they exhibit significant photoactivation when stimulated with blue-light, thus hindering their combination with blue and green-activated opsins ${ }^{21}$. Indeed, most all-optical systems exploiting single-photon excitation critically suffer for crosstalk between imaging and photostimulation 19,21,26,27. To assess the possible cross-activation of ChR2 during wide-field imaging of jRCaMP1a, we performed local field potential (LFP) recordings during an alternating on/off pattern of the imaging illumination path (supplementary fig. 1a). Illumination-triggered average of the LFP showed no significant differences in the normalized power content of the standard neurophysiological spectral bands (supplementary fig. 1b). This result suggests that there were not relevant alterations of the 
neuronal activity during imaging. Therefore, we evaluated whether the laser wavelength used for optogenetic stimulation affected the jRCaMP1a readout. Single laser pulses at increasing intensity were delivered in mice expressing $\left(\mathrm{jRCaMP} 1 \mathrm{a}^{+} / \mathrm{ChR} 2^{+}\right)$or lacking the optogenetic actuator (jRCaMP1 $\mathrm{a}^{+} / \mathrm{ChR2}$ ) (supplementary fig. 1c). The results showed a clear asymptotic increase of the jRCaMP1a response in jRCaMP1a $/ \mathrm{ChR} 2^{+}$mice. Conversely, laser pulses in jRCaMP1 $\mathrm{a}^{+} / \mathrm{ChR} 2^{-}$mice did not induce jRCaMP1a responses up to $20 \mathrm{~mW}$ (supplementary fig. 1c). the results demonstrate that our all-optical configuration wards off the crosstalk between imaging and photostimulation.

\section{Wide-field Imaging during light-based motor mapping reveals the presence of an activation threshold for complex movement execution}

To map forelimb multi-joint movements, we performed optogenetic stimulation of several sites in the motor cortex identifying the locomotion-like movement (TAP; supplementary video I) and the grasping-like movement (GRASP; supplementary video II), (fig. 1a). To establish the movement-specific light-based motor maps (LBMMs), we initially stimulated the previously reported stereotaxic references for the Rostral Forelimb Area (RFA; $+2 \mathrm{~mm} \mathrm{AP},+1.25 \mathrm{~mm}$ LM) and the Caudal Forelimb Area (CFA; $+0.25 \mathrm{~mm} \mathrm{AP},+1.5 \mathrm{~mm} \mathrm{LM})$, in order to induce GRASP and the TAP movements respectively (fig. 2a) ${ }^{4,9,28}$. Stimulus trains at increasing laser power were used to identify the threshold required to elicit a clear motor behavior withinsubjects (fig. 2c-d). The laser pawer thresholds were then employed to design the GRASP and TAP LBMMs (fig. 2b). As previously reported, we found that GRASP and TAP LBMM stereotaxic references were centered in the RFA and CFA respectively ${ }^{4,14}$. The large range of power thresholds (1.3-13.2 mW), could be ascribed to both biological and ChR2 expression variability between subjects. On the contrary, the evoked calcium transients amplitude, that reflects the neuronal ensemble activation, showed limited variability among several animals (GRASP $\Delta \mathrm{F} / \mathrm{F}_{\text {peak }}=15.5 \pm 1 \%$; TAP $\Delta \mathrm{F} / \mathrm{F}_{\text {peak }}=12.7 \pm 1 \%, \mathrm{n}=11$; fig. $2 \mathrm{e}$ ). These observations were further confirmed by the lack of a significant relationship between the stimulus intensities and the amplitude of the evoked calcium transients (fig. 2f). These results suggest a critical activation threshold for triggering complex movement execution.

\section{Movement-specific cortical functional connectivity is bounded to discrete modules}

We wanted to clarify the intrinsic connections and reciprocal functional role of the motor cortices. To this aim, we analyzed the LBMM and the related cortical activation map for each movement category. First, we evaluated the calcium transients evoked in RFA, CFA and nomovement-evoking sites (supplementary fig. 2). The results showed no significant differences between RFA and CFA calcium transient amplitudes (supplementary fig. 2a), while both these areas showed a significantly higher peak amplitude compared with the surrounding nomovement-evoking sites (supplementary fig. $2 \mathrm{~b}$ ). This result suggests a stronger network activation in the movement-evoking areas compared to sites where no macroscopic movements were stimulated. To study the spatial distribution of the activated areas, we performed Maximum Intensity Projections (MIPs) of the cortical imaging stacks recorded during light-based motor mapping (fig. 3a). We defined the movement-specific activation map (MSAM) as the difference between the MIPs obtained within the LBMM boundaries and those obtained close outside those areas (supplementary fig. 2). Subsequently, to investigate the connectivity of the engaged cortical regions we analyzed the LBMM and MSAM spatial profiles (fig. 3a). The results showed that the MSAM area dimension and center of mass were 
comparable to the related LBMM (fig. 3b-c). Moreover, we evaluated the matching of the MSAM with the relative LBMM as the overlap between the maps of the two movement classes. The results showed that RFA and CFA activation networks (MASMs) were clustered in modules overlapping the respective movement cortical topography (LBMMs). Interestingly, RFA and CFA presented limited common activated areas as demonstrated by the low overlap of their MSAMs (fig. 3d and e). These results showed that during movement stimulation there was a large cortical activation confined to the relative movement representation area.

\section{Identification of the Lateral Forelimb Area (LFA) as a distinct grasping representation module}

Although optogenetically-evoked grasping-like and locomotion-like movements were elicited in areas corresponding to the RFA and CFA respectively, we observed that the GRASP LBMM was laterally and caudally stretched beyond the RFA (fig. $2 b$ and $3 a$ ). In half of the examined subjects, this extended GRASP LBMM was separated into two distinct areas one frontal that matched the RFA and the other more caudal and closer to the lateral border of the CFA. We named this area the lateral forelimb area (LFA) (fig. 4a). Initially, we observed that LFA and RFA evoked calcium transients were similar (supplementary fig. 5), evidencing a comparable local neuronal response to the optogenetic stimulus. To understand whether the LFA represented a distinct module or it was an extension of the RFA network, we analyzed the LFA connectivity and its relation with the RFA and CFA. In animals presenting a single extended grasping representation area, we considered its caudal and lateral part as the GRASP LFA (see methods). Our results showed that, as for RFA and CFA, the LFA presented a clear matching of its LBMM and MSAM and their centers of mass were drastically separated from the others (fig. $4 b$ and d). Remarkably, the limited overlap of the LFA MSAM with the maps of the other modules maps reinforced the hypothesis of the discrete functional area (fig. $4 e-f)$. Interestingly, we observed a modest overlap between the MSAM over the LBMM of the LFA (fig. 4d), indicating wider connectivity that exceeded the LBMM borders of the LFA reaching distant areas (fig. $4 \mathrm{a}$ ). These results show that the LFA is associated with specific cortical connectivity features different from that of RFA or CFA.

\section{Grasping-like behaviors evoked in RFA and LFA exhibit similar kinematic profiles}

At a glance, GRASP RFA and GRASP LFA movements presented similar profiles showing an initial forelimb displacement towards the midline followed by elevation to the mouth, which was often coupled with forepaw twisting and licking (fig. 2a; supplementary video II, III). Therefore, to examine in detail their trajectories we tracked the contralateral forelimb movements and performed kinematic analysis. Although GRASP LFA average trajectory was slightly wider compared to GRASP RFA (fig. 5c-d), the absolute maximum lateral displacement and elevation were similar (fig. 5e-f). Moreover, the movement onset time did not show a significant difference between all movement categories (fig. 5b). Conversely, TAP movements displayed completely different trajectories compared to GRASP. Indeed, the locomotion-like movement is rhythmic whereas the grasping-like is a discrete movement. TAP kinematics showed periodicity (fig. 5d), narrow medial-lateral displacement (fig. 5e) and weak elevation (fig. 5f). As expected, the stimulation of no-movement-evoking sites resulted in a remarkably reduced and non-specific forelimb displacement (fig. $5 e-f$ ). Overall, the similarity between GRASP RFA and GRASP LFA kinematics suggests that both cortical modules evoke the same movement. 


\section{Cortical activity propagation analysis reveals movement-specific spatiotemporal patterns of activation}

The spatial analysis highlighted that the optogenetically-evoked complex movements were associated with discrete modules of cortical activation. To investigate the spatiotemporal progression of this activation through cortical areas, we computed the cortical activity propagation map by ranking the time of activation of each pixel in the FOV (fig. 6, see also Methods). From a global (i.e., across the different animals) analysis of these maps, we obtained four polar plots (one for each stimulation condition) describing the spatial propagation direction. Results showed that during RFA stimulation there was a rapid activation of the area around the site of stimulus (green color, fifth rank or earlier) followed by a laterocaudal activation flow that largely preserves its spatial orientation, as shown in fig. 6a. A specular rostromedial flow of activation was observed during CFA stimulation (fig. 6b). Interestingly, LFA stimulation evoked a more complex pattern of cortical activation and the analysis pipeline hardly provides a clear direction for the activation flow (fig. 6c). In addition, no-movementevoking stimulation sites showed a slower spread of activation compared with the complex movement-evoking sites, coupled with an unclear direction of propagation (fig. 6d). Overall, these results reveal that different complex movements are linked with specific spatiotemporal patterns of activity propagation. Interestingly, LFA showed peculiar propagation features, thus reinforcing the hypothesis that LFA relates to a specific grasping evoking module. In addition, the stimulation of movement-related cortical areas leads to a more marked flow of activation compared to no-movements-evoking areas, suggesting the persistence of more complex connectivity associated with movement execution.

\section{Excitatory synaptic block leads to disruption of connectivity features associated with complex movement interference}

The spatiotemporal analysis suggested a progressive engagement of specific regions in the motor cortex. A common strategy to dissect the role of different functional nodes in a neuronal network is the pharmacological synaptic transmission block, in particular using glutamatergic transmission antagonists ${ }^{14,29,30}$. To investigate both the role of the local connectivity and the reciprocal role of each module during optogenetic-evoked movement execution, we performed a module-specific block of the excitatory synaptic transmission through topical application of the AMPA/kainate receptor antagonist 6-cyano-7-nitroquinoxaline-2,3-dione (CNQX) on the cortical surface ${ }^{14,30,31}$. The results showed that CNQX application in RFA reduces the extension of the GRASP RFA activation map (fig. 7b) while it does not significantly affect the calcium transient profiles (fig. 7c). Similar results were obtained by applying CNQX in CFA (fig. $7 \mathrm{~b}-\mathrm{c}$ ). These results suggest an impairment of the local connectivity (a decrease in activation spreading) that does not affect the direct local response to the optogenetic stimulus. These results are in accordance with evidence showing that topical application of CNQX disrupts the cortical connectivity while preserving the direct activation of ChR2-expressing neurons ${ }^{14}$. Moreover, to analyze the effect of the module-specific block of the excitatory synaptic transmission on the activity propagation features, we compared the pixel rank distribution of an ROI overlapping the relative LBMM before and after the pharmacological block (fig. 7d-e). These results highlight an increase in both the median and the interquartile range (IQR) caused by the pharmacological connectivity interference suggesting a slower and more disorganized propagation of the cortical activity, respectively (fig. $7 \mathrm{f}$ ). It should be noted that these results correlate with the behavioral outcome (fig. $7 \mathrm{~g}$ ). Indeed, as previously 
reported by Harrison et al., 2012, CNQX application leads to faults and distortions in complex movement execution, until the complete extinction of a recognizable complex movement ${ }^{14}$. Interestingly, CNQX application in RFA resulted in a block of GRASP execution while preserving the CFA-evoked TAP movement. The same result was obtained following the application of CNQX in CFA that resulted in a block of the TAP while maintaining a successful GRASP RFA expression. Taken together these results demonstrate that local excitatory synaptic inputs are required to control complex motor behaviors, and that GRASP RFA and TAP are controlled by two independent cortical modules.

\section{LFA-evoked grasping does not require RFA activation}

Connectivity analysis suggested that the three identified functional modules activate distinct cortical regions during movement execution and the excitatory synaptic block experiments confirmed that GRASP RFA and TAP do not require mutual activation (fig. 7). In order to further study the relation between LFA and RFA, we stimulated the LFA during RFA pharmacological block (fig. 8). The results showed that there was no significant difference neither in the LFA MSAM dimension (fig. 8b) nor in evoked-calcium transients (fig. 8c). Moreover, we observed that the RFA pharmacological block did not modify the LFA spatiotemporal propagation features (fig. 8d-e). Interestingly, the preserved cortical connectivity of the LFA correlated with the successful execution of the GRASP LFA movement despite the block in RFA (fig. 8f). These results demonstrate that the LFA motor output does not require the RFA activation. 


\section{DISCUSSION}

Exploiting a large-scale all-optical method, we mapped the RFA and CFA activity during stimulated complex movement execution. Thanks to our novel approach we provided evidence for a segregated organization of RFA and CFA networks. The areas engaged during RFA and CFA stimulation were clustered over their optogenetic maps and did not overlap. Moreover, the spatiotemporal activity propagation analysis reveals movement-specific patterns. The pharmacological disruption of RFA or CFA inhibited the execution of the related movement while preserving the behavioral output and the activation features of the other areas, demonstrating their partitioned functional structure. Remarkably, we identified a second grasping representation area, functionally independent from the RFA and expressing distinct activation features, that we named Lateral Forelimb Area (LFA).

To causally investigate neuronal circuits, optogenetics has been paired with single- and twophoton fluorescence imaging in all-optical neurophysiology approaches ${ }^{19,32}$. There are still important limitations matching optogenetics and fluorescence imaging due to the large crosstalk between functional indicators and optogenetic actuators, caused by their spectral overlap. This phenomenon leads to spurious modulation of neuronal activity during imaging or stimulation artifacts in the readout channel, making it hard to develop a cross-talk free alloptical method ${ }^{19,33,34}$. Recently, Forli et al. took advantage of two-photon absorption for both jRCaMP1a excitation and ChR2 activation to manipulate and record cortical neuronal activity in anesthetized mice demonstrating this solution as the best option to drastically reduce crosstalk ${ }^{23}$. Here we extended this configuration to transcranial single-photon excitation in awake head-fixed mice. We initially evaluated the cross-activation of jRCaMP1a and ChR2 using visible light excitation. The electrophysiological analysis showed that the imaging excitation did not affect the LFP content, suggesting that there is no detectable neuronal activation during jRCaMP1a imaging (supplementary fig. 1a-b). This result is in line with previous evidence showing the absence of crosstalk using RCaMPs and ChR2 ${ }^{21-23}$. In particular, using the patch-clamp technique, it has been demonstrated that one-photon widefield illumination at $590 \mathrm{~nm}$ of cultured neurons expressing ChR2 prevents photocurrent generation, thus maintaining subthreshold potentials ${ }^{23}$. The second aspect we considered was the jRCaMP1a activation following blue-laser excitation. As shown in supplementary fig. $1 \mathrm{c}$ the laser stimulation did not change the jRCaMP1a fluorescence dynamics in mice lacking $\mathrm{ChR} 2$, demonstrating that optogenetic stimulation did not affect the neuronal activity readout. Besides, we optimized a transfection strategy to achieve a wide and stable expression of both the optogenetic actuator and the fluorescence reporter over one hemisphere, exploiting a double AAV viral vector injection. Consistently with our previous study ${ }^{20}$, we obtained a stable expression for both jRCaMP1a and ChR2 in the right hemisphere motor cortex (fig. 1). It should be noted that we did not observe bleaching of jRCaMP1a signal after prolonged exposure to our imaging sessions and after dozens of consecutive optogenetic stimulations (supplementary fig. 3; see methods). However, our method lacks optical sectioning, thus it is hard to control either the cortical layers targeted by the optogenetic stimulation or the exact source of the fluorescence signal given the spatial profile of the jRCaMP1a expression (fig. 1d). Therefore, further studies will be necessary to clarify these points.

Due to technical limitations, investigations of the cortical connectivity related to natural behaviors ${ }^{17,18}$ and motor mapping studies ${ }^{4,35}$ are largely confined to separated experiments. Previous research showed that a rich repertoire of complex movements can be evoked optogenetically-stimulating different sites in the mouse motor cortex ${ }^{4,14}$. In the present study, 
we developed a method to simultaneously analyze the cortical representation of complex movements and the related features of mesoscale activation. We focused on two forelimb movements, the discrete forepaw-to-mouth movement (GRASP) and the rhythmic locomotionlike movement (TAP) obtained by stimulating the RFA and CFA respectively ${ }^{4,12,13}$. According to the literature, the quantitative kinematic analysis revealed that the TAP trajectory exhibited rhythmic repetitions and a slight lateral displacement while the GRASP trajectory was mainly displaced in the mediolateral plane and exhibited a discrete elevation of the forelimb toward the mouth (fig. 5) ${ }^{4,14}$. Nevertheless, the observed movements were characterized by larger onset times compared to the literature, this discrepancy could be ascribed to both the lower stimulation frequency and the different animal models used.

In order to map the cortical topography of these movements, we evaluated the minimum laser power required to elicit a clear GRASP or TAP by gradually increasing the stimulus intensity. Results showed variable subject-specific power values. Interestingly, the related cortical activation exhibited lower between-subjects heterogeneity (fig. 2). This difference could be ascribed to the between-subjects variability in the opsin expression. Indeed, the efficiency of the optogenetic stimulation depends on the absolute protein expression whereas the imaging signal is normalized as relative fluorescence changes $(\Delta F / F)$, which reduces the impact of the expression variability on the readout. Once the subject-specific minimum laser power was identified, we designed the GRASP and TAP LBMMs. In accordance with previous studies, we found that GRASP and TAP LBMMs covered the RFA and CFA respectively (fig. 2b) ${ }^{4,14}$. Our novel all-optical tool allowed us to study the amplitude of the calcium transients evoked in GRASP and TAP LBMMs that were significantly different from those evoked in nomovement-evoking areas (Supplementary fig. 3b). This result suggests stronger intrinsic connectivity of the complex movement representation areas. Moreover, our spatial analysis revealed that the GRASP representation extended laterally beyond the RFA towards the forelimb somatosensory (FLS1) cortex (fig. $2 b$ and $3 a$ ). Therefore, we characterized this lateralization identifying the LFA (fig. 4). Previously Bonazzi et al. mapped the motor cortex topography in anesthetized rats through ICMS, describing a lateral area expressing a holdlike forelimb movement, defined as the paw supination (the wrist and forearm turning toward the midline or the face) ${ }^{10}$. In the lateral part of the rat motor map, the authors showed that the hold-like movement is coupled with elevation and abduction movements that resemble the feature that we considered for grasping movement. Interestingly, also Harrison et al. exploiting the light-based motor mapping technique, observed a lateral extension of the forelimb abduction movement centered in the RFA. Moreover, previous studies reported that corticospinal motor neurons (CSN) can be found in RFA, CFA, and in a small circumscribed cluster in the secondary somatosensory cortex named PL-CFA ${ }^{36,37}$. At the spinal cord level, CSN axons from PL-CFA mainly overlap with the RFA-CSNs premotor neurons ${ }^{36,38}$, suggesting the control of the same group of muscles. Therefore, the LFA that we functionally characterized in this paper could refer to the group of neurons anatomically described in these previous works. Optogenetic stimulation of RFA and LFA lead to the generation of similar grasping behavior, therefore, we investigated the functional role of the LFA compared to the RFA, starting with the kinematic analysis of the respective movements. The results showed that GRASP RFA and GRASP LFA expressed comparable trajectories achieving equal elevation and displacement in the medial-lateral plane and displaying the same onset time (fig. 5). This result confirms that LFA and RFA exhibit the same motor output.

To study the connectivity hallmarks of GRASP RFA, GRASP LFA and TAP, we calculated the movement-specific activation maps (MSAMs). We found that the MSAMs were packed in segregated modules that largely overlapped the associated movements representation 
topography (fig. 3 and 4). Indeed, GRASP RFA topography and MSAM circumvent the TAP area and the relative MSAM. The same results were observed for the TAP movement. Remarkably, also the LFA activation map avoided the RFA- and CFA-relative LBMMs and MSAMs, displaying specific connectivity features (fig. 4). ICMS stimulation has been recently coupled with intrinsic signal optical imaging to reconstruct activation maps related to forelimb stimulated movements in squirrel monkeys ${ }^{39}$. The authors demonstrated that the intrinsic motor cortex connectivity matched the forelimb somatotopic representation in the primary motor cortex ${ }^{39}$. Our results are in line with evidence suggesting a segregated functional organization of CFA and RFA ${ }^{4,11}$, despite their mutual connections that may have a role in coordinating sequences of complex movements such as the reach to grasp behavior ${ }^{40,41}$. Interestingly, the spatial clustering of functionally correlated units in the motor cortex seems to be expressed across scales from neurons ${ }^{42}$ to entire functional areas ${ }^{4}$.

Spatiotemporal activity propagation features are pivotal aspects of the computation and communication between subsystems of the brain ${ }^{43}$. In the motor cortex, behaviorally relevant propagating patterns of cortical activation have been demonstrated to be necessary for movement initiation ${ }^{44,45}$. Therefore, we explored the spatiotemporal spreading of the neuronal activity during stimulated motor performances and we found movement-specific propagation patterns for the three motor regions (fig. 6). Our analysis revealed movement-specific orientation of the activity propagation, showing opposite directions for RFA and CFA. Conversely, LFA patterns exhibited more complex features rather than the fairly linear propagation observed for the other modules. These results reinforce the idea that LFA could represent a distinct GRASP representation.

To test the correlation between the simulated movements and the activity features observed, we performed module-specific inhibition of the excitatory synaptic transmission. This pharmacological tool allows an effective direct optogenetic stimulation of the targeted area while blocking its input connections, thus probing the role of the module-specific network in generating the forelimb movement. It has been demonstrated that topical application of the AMPA/kainate receptor antagonist CNQX on the cortical surface disrupts the optogeneticevoked complex movement execution while preserving the direct activation of ChR2expressing neurons ${ }^{14}$. Accordingly, our results show that the RFA pharmacological inactivation interferes with the GRASP execution while retaining the ability to evoke the TAP movement and the specular phenomenon was observed during CFA inhibition (fig. 7), supporting the idea of two functionally independent modules ${ }^{4,14}$. Moreover, we reported that CNQX application leads to a significant reduction of the MSAM extension associated with slower and more disorganized patterns of local propagation (fig. 7), highlighting that the activation features we described reflect the module-specific network activity linked to movement execution. These results sharpen the idea that direct activation of corticospinal projections is not sufficient to stimulate full movement performance, thus confirming the pivotal role of the cortical synaptic inputs. Further studies will be necessary to understand the contribution of the recurrent cortico-cortical circuits ${ }^{46,47}$ or subcortical loops ${ }^{48}$ in complex movement control and to define whether the modules found in the motor cortex and their related inputs can be organized as central pattern generator networks, in which the activation of a group of neurons can be sufficient to elicit an entire motor engram ${ }^{49,50}$. Moreover, we observed that during RFA inactivation the LFA behavioral output and all its activation features were preserved (fig. 8), demonstrating that the GRASP LFA expression is not affected by the RFA network, thus suggesting that the two grasping modules are parallelly organized.

To the best of our knowledge, this is the first application of a large-scale all-optical method in awake mice. The experimental paradigm we developed represents a powerful approach to 
causally dissect the cortical connectivity, reaching its full potential in experimental settings where it is not possible to record behavioral outputs, for instance in the study of non-motor cortical regions or the investigation of different brain states and pathologies with altered level of consciousness i.e., sleep, anesthesia or coma ${ }^{51}$. Exploiting this method, we raised evidence for a segregated functional organization of CFA and RFA and we identified a new forelimb representation area. Further studies will be necessary to define the ethological role of the LFA and its engagement in voluntary movements. 


\section{METHODS}

Virus injection and intact-skull window. All experiments were performed in accordance with the guidelines of the Italian Minister of Health (aut. n. 871/2018). C57BL/6J adult mice (6-12 months) of both sexes were anesthetized with isoflurane (3\% for induction, $1-2 \%$ for maintenance) and placed in a stereotaxic apparatus (KOPF, model 1900). Ophthalmic gel (Lacrilube) was applied to prevent eye drying, body temperature was maintained at $36^{\circ} \mathrm{C}$ using a heating pad and lidocaine $2 \%$ was used as local anesthetic. The skin and the periosteum were cleaned and removed. Bregma was signed with a black fine-tip pen. To achieve widespread expression of both $\mathrm{jRCaMP1a}$ and $\mathrm{ChR} 2$ over the right hemisphere, small holes were drilled at two coordinates (AP $+2.0 \mathrm{~mm}, \mathrm{ML}+1.7 \mathrm{~mm}$; $A P-0.5 \mathrm{~mm}, \mathrm{LM}+1,7 \mathrm{~mm}$ from bregma). A $500 \mathrm{nl}$ volume of mixed viruses (pGP-AAV9-syn-NES-jRCaMP1aWPRE.211.1488 and pAAV9-CamKII-hChR2(H134R)-Cerulean, $1 \times 10^{13}$ GC $\mathrm{ml}^{-1}$, CliniSciences, $250 \mathrm{nl}$ respectively) was pressure-injected through a pulled glass micropipette at one depth per site $(-0.5 \mathrm{~mm}$ ventral from dura surface) using an electrically gated pressure injector (Picospritzer III-Science Products ${ }^{\mathrm{TM}}, \mathrm{n} 3 \mathrm{~Hz}$, ON $4 \mathrm{~ms}$ ) for a total volume of $1 \mu \mathrm{l}$ per mouse. A custom-made aluminum head-bar placed behind lambda and a cover glass implanted on the exposed skull were fixed using transparent dental cement (Super Bond C\&B - Sun Medical). After the surgery, mice were recovered in a temperature- and humiditycontrolled room, with food and water ad libitum for two weeks before recordings.

Wide-field microscopy setup. Wide-field imaging and optogenetic stimulation were performed using a custom-made microscope with two excitation sources to simultaneously excite the opsin (ChR2-cerulean) and the calcium indicator (jRCaMP1a) ${ }^{52}$. The excitation source for jRCaMP1a was a red-light beam of emitting diodes (595nm LED light, M595L3 Thorlabs, New Jersey, United State) and the excitation band was selected by a bandpass filter (578/21 nm, Semrock, Rochester, New York, USA). The light beam was deflected by a dichroic mirror (606nm, Semrock, Rochester, New York, USA) to the objective (2.5x EC Plan Neofluoar, NA 0.085) towards the skull. The excitation source for single-photon stimulation of ChR2 was a continuous wavelength (CW) laser $(\lambda=473 \mathrm{~nm}$, OBIS $473 \mathrm{~nm}$ LX 75mW, Coherent, Santa Clara, CA, USA). The excitation beam was overlaid on the imaging pathway using a second dichroic beam splitter (FF484-Fdi01-25 × 36, Semrock, Rochester, New York, NY, USA) before the objective. The system has a random-access scanning head with two orthogonallymounted acousto-optical deflectors (DTSXY400, AA Opto-Electronic, Orsay France). The jRCaMP1a fluorescence signal emitted was collected through a band-pass filter (630/69, Semrock, Rochester, New York, USA) and focused by a tube lens (500 nm) on the sensor of a demagnified (20X objective, LD Plan Neofluar, 20×/0.4 M27, Carl Zeiss Microscopy, Oberkochen, Germany) high speed complementary metal-oxide semiconductor (CMOS) camera (Orca Flash 4.0 Hamamatsu Photonics, NJ, USA). The camera acquired images at a resolution of 100 by 100 pixels covering a quadratic field-of-view of 5.2 by $5.2 \mathrm{~mm}^{2}$ of the cortex.

Wide-field imaging in awake mice. 14 days after the injection, head-fixed imaging sessions were performed for three consecutive weeks. An animal-specific field of view (FOV) template was used to manually adjust the imaging field daily. Each imaging session consisted of 5-10 $\mathrm{s}$ of recording in resting-state followed by the stimulus train (2s) and $30 \mathrm{~s}$ of imaging after the stimulus (sampling rate: $50 \mathrm{~Hz}$ ). The waiting time for consecutive sessions was 3 minutes per animal. LED light intensity was $4 \mathrm{~mW}$ after the objective. 
Transcranial optogenetic stimulation. Laser stimulation patterns were generated using two orthogonally-mounted acousto-optical deflectors controlled by a custom-written LabView 2013 software (National Instruments). A reference image of the FOV was used to target the laser beam on a selected cortex area.

Single-pulse laser stimulation consisted of one pulse $(10 \mathrm{~ms}$ ON) repeated 8 times in one imaging session at different laser power $(0.22-1.3-2.5-5.2-7.7-13.2 \mathrm{~mW}$, after the objective).

The stimulus train consisted of $2 \mathrm{~s}, 16 \mathrm{~Hz}, 10 \mathrm{~ms} \mathrm{ON}$. For laser power calibration experiments the laser power used were: 1,3- 2,5 - 5,2 - 7,7 - 13,2 mW. For light-based motor mapping, connectivity studies and pharmacological inhibition laser power was the minimum power required to evoke movements (from $1.3 \mathrm{~mW}$ to $13.2 \mathrm{~mW}$ ).

Light-based motor map (LBMM). The LBMMs for locomotion-like (TAP) and grasping-like (GRASP) movements were obtained in separate experiments. A virtual grid (14 x 14, $364 \mu \mathrm{m}$ spacing) was superimposed on the animal-specific FOV template using Fiji ${ }^{53}$. A stimulus train was then delivered in a random order single time for all sites of the grid. The left forepaw position during imaging sessions was monitored using a camera equipped with a red illumination light focused on the forepaw and not interfering with imaging. Forelimb movements were evaluated by two different expert observers and visually categorized as (i) grasping-like movements: contralateral forepaw was closed, the wrist turned and moved toward the mouth (ii) locomotion-like movements: contralateral forelimb was retracted and lifted at least twice, simulating a walking movement (iii) no-movements and movement interference: the absence of at least one movement criterion during the stimulus. The average LBMM was created by aligning three points in the FOV (bregma and injection sites) in 7 animals per movement category.

Optogenetic of the Lateral Forelimb Area (LFA) LBMM. 4 out of 8 animals presented a discrete LFA and RFA light-based motor map. The average of these LFA LBMM, with a $100 \%$ threshold (total overlap for high restriction), was used as a mask for identifying the LFA border in those animals that presented a unified light-based motor map.

Video tracking analysis. A machine vision camera (PointGrey flir Chamaleon3, CM3-U313Y3C-CS) was orthogonally set $100 \mathrm{~mm}$ in front of the mouse to evaluate the left forelimb movement induced by contralateral optogenetic stimulation (frame rate $100 \mathrm{~Hz}$ ). The camera acquired images at a resolution of 800 by 600 pixels covering a field-of-view of 24 by $18 \mathrm{~mm}^{2}$ of the cortex (0.03 $\mathrm{mm}$ per pixel). Visible illumination light at $630 \mathrm{~nm}$ was focused on the left forepaw, to avoid imaging interference. Five individual trains per movement category for each animal were filmed $\left(n_{\text {mice }}=5 ; n_{\text {trains }}=5\right)$. Videos were analyzed using the ImageJ plugin AnimalTracker, obtaining $X Y$ coordinates of the forelimb in each frame from a starting point (Ref. ${ }^{54}$ for details). To compare evoked complex movements, we analyzed the tracked forelimb mediolateral displacement, the elevation and the speed $(\mathrm{mm} / \mathrm{s})$ for each train.

In-vivo local field potential recording. Local field potentials (LFPs) were recorded in the center of the transfected area. Glass pipettes were used to avoid light-induced artifacts during the electrophysiological recordings and were filled with a $2 \mathrm{M} \mathrm{NaCl}$ solution. The electrode was advanced, through a little hole in the skull, into the motor cortex L5 (800 $\mu \mathrm{m}$ from the dura surface) using a motorized micromanipulator (EXFO Burleigh PCS6000 Motorized 
Manipulator). Signals were amplified with a $3000 \mathrm{AC} / \mathrm{DC}$ differential amplifier, sampled at 10 $\mathrm{kHz}$, highpass filtered at $0.1 \mathrm{~Hz}$ and lowpass filtered at $3 \mathrm{kHz}$. A reference and ground screws were placed on the occipital bone. LFP signal was recorded during a randomly activated pattern of led ON / led OFF (2 seconds each, $4.5 \mathrm{~mW}$ ). As a control, an optogenetic singlepulse stimulus was delivered close to the pipette tip, resulting in a fast downward deflection, indicating that $\mathrm{ChR} 2$ was effectively transfected and functioning.

Preprocessing of imaging data. Images were analyzed with ImageJ and OriginPro (OriginLab 2017). Frames displaying artifactual excitation of skull autofluorescence were removed (2 out of 3 frames) and interpolated. A daily individual mask was created using the maximum intensity projection of the first imaging session (baseline). Masks were thresholded twice the mean value of the non-transfected hemisphere. For each imaging session, the fluorescence ratio change $\left(\Delta \mathrm{F} / \mathrm{F}_{0}\right)$ was calculated averaging the first 50 frames before the stimulus onset (baseline fluorescence signal; $F_{0}$ ).

Calcium data analysis. In vivo quantification of jRCaMP1a and ChR2 spatial distribution. The full width at half-maximum (FHWM) of spatial fluorescence profile in vivo was evaluated during the third and fourth weeks after injection. FWHM was calculated on the average of the three brightest frames acquired in the resting state imaging session, over two parallel lines that crossed the injection sites in the mediolateral plane.

Single-pulse correlation was performed during the third and fourth weeks after injection. Single-pulse laser stimulations were delivered to the cortex region with the maximum level of ChR2 and jRCaMP1a expression. The consequently evoked calcium response dynamics (time series) were extracted from a region of interest (ROI, area $0,24 \mathrm{~mm}^{2}$ ) placed over the stimulation site.

Power calibration. The optogenetic stimulus was delivered in the center of the light-based motor map. The stimulus train was repeated 3 times at increasing laser power to select the minimum power required to evoke GRASP and TAP movements. Calcium dynamics (time series) were extracted from a ROI (area $0,24 \mathrm{~mm}^{2}$ ) placed over the site of stimulation.

Movement specific calcium map. An imaging stack was recorded for each site of the grid stimulated during the light-based motor mapping. For each acquisition $(14 \times 14,364 \mu \mathrm{m}$ spacing), the Maximum-Intensity Projection (MIP) was obtained and subsequently grouped by evoked-movement category in (i) GRASP RFA movement, (ii) GRASP LFA movement, (iii) TAP movement. Since light-based motor maps for TAP and GRASP were obtained in separate experiments, there were two different groups of no-movement: (iv) GRASP-related nomovement and ( $v$ ) TAP-related no-movement. An average maximum activity value based on all MIPs was then calculated for GRASP RFA/LFA and TAP categories, and half of that value has been used for thresholding the MIPs of all groups. Thresholded MIPs were averaged and an additional threshold of $2 x$ standard deviation (SD) was applied, obtaining the average activation map for all five experimental groups. Finally, in order to obtain movement-specific activation maps (MSAMs), the non-specific movement average activation maps were spatially subtracted from those related to GRASP RFA, GRASP LFA and TAP. The spatial overlap between the MSAM and the related LBMM was then assessed and quantified as a percentage of the total dimension of both the maps involved.

Spatiotemporal propagation analysis. Spatiotemporal propagation analysis was performed with custom-made Python (Python Software Foundation, Beaverton, Oregon, U.S.A.) scripts. 
In a pre-processing step, image sequences were spatially masked and frames containing the laser stimulations were manually eliminated and replaced with their temporal linear interpolation. Then a Gaussian smoothing was performed along the temporal dimension (with a standard deviation for Gaussian kernel equal to one) before computing the $\Delta F / F_{0}$ signal. $F_{0}$ was set as the average fluorescence value observed before the first laser stimulus. Pixels were identified as active if the maximum value of the $\Delta \mathrm{F} / \mathrm{F}_{0}$ signal after the first laser stimulus was larger than both the average value and the double of the standard deviation value, computed in both cases before the first stimulus. In active pixels, the time frame corresponding to the first crossing of a pixel-based threshold was used to identify the timing of the response to the laser stimulus. The threshold was set as twice the standard deviation value of the $\Delta F / F_{0}$ signal computed before the first laser stimulus. For imaging acquisitions pertaining to CNQX manipulation, the standard deviation values used to identify the active pixels and to define the timing thresholds were computed solely on the data acquired before CNQX administration (vehicle). For the data acquired after CNQX administration, the average of the standard deviations computed before CNQX administration was employed. In all cases, the timing values were then rank-transformed. The rank values of the active pixels related to the same animal and the same condition were averaged and the standard deviation was computed, while the non-active pixel values were discarded. Starting from these averaged results, for data related to CNQX manipulation, rank distributions were computed in a region of interest (ROI) overlapping the LBMMs. The distribution medians before and after CNQX administration were then compared using Wilcoxon signed-rank test. Moreover, to summarize the distribution characteristics, their interquartile ranges were computed alongside the medians. Finally, to trace the propagation direction, for each averaged result, pixels placed along a circumference centered on the laser-stimulated area and with varying radius were selected, discarding nonactive or masked pixels. For each circumference radius, the averaged rank distribution was computed and values composing its first quintile were sub-selected. Then the circular mean ${ }^{55}$ of the angular position (relative to the circumference center) of these values was computed. Finally, all the computed circular means were used to calculate the final, radius-dependent, circular mean and circular standard deviation ${ }^{55}$.

Pharmacology. For pharmacological interference experiments, a small craniotomy $(1 \mathrm{~mm}$ diameter) was performed on the region of interest. For the animals that underwent pharmacological inhibition of both GRASP and TAP, the experiments were performed in a 3day separate section. The craniotomies were then sealed with Kwik-seal (World Precision Instrument) after the experimental sections. Glutamate receptor antagonist CNQX $1 \mathrm{mM}$ (C127 Sigma-Aldrich) and vehicle (physiological solution containing $0.01 \%$ DMSO) were applied to the craniotomy and the solutions were replenished (at the same concentration) every 10 ' to compensate for tissue drying. Stimulation sessions were performed every 10'.

Immunohistochemistry. Four weeks after injection, mice were perfused with 20-30 $\mathrm{ml}$ of 0.1 M PBS (pH 7.6) and $150 \mathrm{ml}$ of 4\% paraformaldehyde (PFA). Brain coronal slices (100 $\mu \mathrm{m}$ thick) were cut with a vibrating-blade microtome (Vibratome Series 1500-Tissue Sectioning System). Slices were washed with PBS and incubated in PBS/0.3\% Triton X-100 containing $1 \%$ bovine serum albumin (BSA) for 60 min while shaking at room temperature (RT). Then, slices were washed with PBS $/ 0.1 \%$ Triton X-100 (T-PBS) and incubated with the primary antibody NeuN (1:200, Sigma, ABN78) in T-PBS for 1 day at $4^{\circ} \mathrm{C}$ while shaking. Then, slices were washed with T-PBS and incubated with anti-rabbit fluorescent Alexa 514 antibody $(1: 250$, ThermoFisher, A-31558) in T-PBS for $2 \mathrm{~h}$ at RT while shaking. Finally, slices were washed and mounted on a glass slide. Imaging was performed with a confocal laser scanning 
bioRxiv preprint doi: https://doi.org/10.1101/2021 07.15.452461: this version posted July 15, 2021. The copyright holder for this preprin (which was not certified by peer review) is the author/funder, who has granted bioRxiv a license to display the preprint in perpetuity. It is made available under aCC-BY-ND 4.0 International license.

microscope (CLSM, Nikon Eclipse TE300, with the Nikon C2 scanning head), equipped with a Nikon Plan EPO 60× objective, N.A. 1.4, oil immersion). The setup was equipped with 408 $\mathrm{nm}, 488 \mathrm{~nm}$ and $561 \mathrm{~nm}$ lasers to simultaneously excite ChR2, Alexa 514 and jRCaMP1a, respectively. A triple-band dichroic mirror $408 / 488 / 543$ was used for simultaneous 3-channel fluorescence imaging. Emission filters were $472 / 10 \mathrm{~nm}, 520 / 35 \mathrm{~nm}$ and 630/69 nm.

Statistics. All statistical analysis was performed in OriginLab 2018 except for the spatiotemporal propagation analysis that was performed with custom-made Python (Python Software Foundation, Beaverton, Oregon, U.S.A.) scripts. Data are shown as mean \pm s.e.m. Parametric tests were used only after verifying for normality of the data employing ShapiroWilk and Kolmogorov-Smirnov tests. The error bars and shadows in graphs represent the s.e.m. In the box charts, the red line corresponds to the mean, the box shows the standard error range, whiskers lengths are the extreme data points. Student's t-test was employed for every comparison concerning two samples and its paired version was used for paired data (fig. $7 \mathrm{~b}$ and c; fig. $8 \mathrm{~b}$ and c). For spectral band multiple comparisons in supplementary fig. $1 \mathrm{~b}$, two-way (variables: illumination status and bands) ANOVA was used. For multiple comparisons in Figure 6b,e,f (for onset, distance and elevation values, respectively) and in Supplementary figure $5 \mathrm{a}$ (for average calcium transients), one-way ANOVA was used and Bonferroni correction was applied for post-hoc t-tests. The level of significance was set at ${ }^{*} p$ $<0.05,{ }^{* *} p<0.01$, and ${ }^{* * *} p<0.001$. 
bioRxiv preprint doi: https://doi.org/101101/2021 07.15.452461: this version posted July 15,2021 . The copyright holder for this preprin (which was not certified by peer review) is the author/funder, who has granted bioRxiv a license to display the preprint in perpetuity. It is made available under aCC-BY-ND 4.0 International license.

\section{ACKNOWLEDGMENTS}

This research has been supported by the European Union's Horizon 2020 research and innovation Framework Programme under grant agreements N. 945539 (HBP-SGA3), N. 785907 (HBP-SGA2) and from the EU program H2020 EXCELLENT SCIENCE - European Research Council (ERC) under grant agreement n. 692943 (BrainBIT).

This research has also been supported by the Italian Ministry for Education, University, and Research in the framework of the Advance Lightsheet Microscopy Italian Mode of EuroBioimaging ERIC. 


\section{REFERENCES}

1 Schaal S., Sternard D., Osu R. \& M., K. Rhythmic arm movement is not discrete. Nature Neuroscience 7, doi:10.1038/mm1322 (2004).

2 Degallier, S. \& ljspeert, A. Modeling discrete and rhythmic movements through motor primitives: a review. Biol Cybern 103, 319-338, doi:10.1007/s00422-010-0403-9 (2010).

3 Howard, I. S., Ingram, J. N. \& Wolpert, D. M. Separate representations of dynamics in rhythmic and discrete movements: evidence from motor learning. J Neurophysiol 105, 1722-1731, doi:10.1152/jn.00780.2010 (2011).

4 Hira, R., Terada, S., Kondo, M. \& Matsuzaki, M. Distinct Functional Modules for Discrete and Rhythmic Forelimb Movements in the Mouse Motor Cortex. J Neurosci 35, 13311-13322, doi:10.1523/JNEUROSCI.2731-15.2015 (2015).

5 Morandell, K. \& Huber, D. The role of forelimb motor cortex areas in goal directed action in mice. Sci Rep 7, 15759, doi:10.1038/s41598-017-15835-2 (2017).

6 Neafsey, E. J. et al. The organization of the rat motor cortex: A microstimulation mapping study. 77-96, doi:10.1016/0165-0173(86)90011-1 (1986).

7 Graziano, M. S. A., Taylor, C. S. R. \& Moore, T. Complex Movements Evoked by Microstimulation of Precentral Cortex. Neuron 34, 841-851 (2002).

8 Ramanathan, D., Conner, J. M. \& Tuszynski, H. M. A form of motor cortical plasticity that correlates with recovery of function after brain injury. PNAS 103, 11370-11375, doi:10.1073/pnas.0601065103 (2006).

9 Tennant, K. A. et al. The organization of the forelimb representation of the C57BL/6 mouse motor cortex as defined by intracortical microstimulation and cytoarchitecture. Cereb Cortex 21, 865-876, doi:10.1093/cercor/bhq159 (2011).

10 Bonazzi et al. Complex Movement Topography and Extrinsic Space Representation in the Rat Forelimb Motor Cortex as Defined by Long-Duration Intracortical Microstimulation. Journal of Neuroscience 33, 2097-2107, doi:10.1523/jneurosci.3454-12.2013 (2013).

11 Brown, A. R. \& Teskey, G. C. Motor cortex is functionally organized as a set of spatially distinct representations for complex movements. J Neurosci 34, 13574-13585, doi:10.1523/JNEUROSCI.2500-14.2014 (2014).

12 Ayling, O. G., Harrison, T. C., Boyd, J. D., Goroshkov, A. \& Murphy, T. H. Automated light-based mapping of motor cortex by photoactivation of channelrhodopsin-2 transgenic mice. Nat Methods 6, 219-224, doi:10.1038/nmeth.1303 (2009).

13 Hira, R. et al. Transcranial optogenetic stimulation for functional mapping of the motor cortex. Journal of Neuroscience Methods 179, 258-263, doi: 10.1016/j.jneumeth.2009.02.001 (2009).

14 Harrison, T. C., Ayling, O. G. \& Murphy, T. H. Distinct cortical circuit mechanisms for complex forelimb movement and motor map topography. Neuron 74, 397-409, doi:10.1016/j.neuron.2012.02.028 (2012).

15 Kunori, N. \& Takashima, I. High-order motor cortex in rats receives somatosensory inputs from the primary motor cortex via cortico-cortical pathways. Eur J Neurosci 44, 2925-2934, doi:10.1111/ejn.13427 (2016).

16 Guo, J. Z. et al. Cortex commands the performance of skilled movement. eLife 4, doi:10.7554/eLife.10774 (2015).

17 Makino, H. et al. Transformation of Cortex-wide Emergent Properties during Motor Learning. Neuron 94, 880-890.e888, doi:10.1016/j.neuron.2017.04.015 (2017).

18 Quarta, E. et al. A distributed neocortical action map associated with reach-to-grasp. biorxiv, doi:10.1101/2020.01.20.911412 (2020). 
19 Emiliani, V., Cohen, A. E., Deisseroth, K. \& Hausser, M. All-Optical Interrogation of Neural Circuits. J Neurosci 35, 13917-13926, doi:10.1523/JNEUROSCI.2916-15.2015 (2015).

20 Montagni, E. et al. Wide-field imaging of cortical neuronal activity with red-shifted functional indicators during motor task execution. Journal of Physics D: Applied Physics 52, doi:10.1088/1361-6463/aaf26c (2019).

21 Akerboom, J. et al. Genetically encoded calcium indicators for multi-color neural activity imaging and combination with optogenetics. Frontiers in Molecular Neuroscience 6, doi:10.3389/fnmol.2013.00002 (2013).

22 Dana, H. et al. Sensitive red protein calcium indicators for imaging neural activity. Elife 5, doi:10.7554/eLife.12727 (2016).

23 Forli, A. et al. Two-Photon Bidirectional Control and Imaging of Neuronal Excitability with High Spatial Resolution In Vivo. Cell Rep 22, 3087-3098, doi:10.1016/j.celrep.2018.02.063 (2018).

24 Zhao, Y. et al. An Expanded Palette of Genetically Encoded Ca2+ Indicators. Science 333, 1888-1891, doi:10.1126/science.1208592 (2011).

25 Farhi, S. L. et al. Wide-Area All-Optical Neurophysiology in Acute Brain Slices. J Neurosci 39, 4889-4908, doi:10.1523/JNEUROSCI.0168-19.2019 (2019).

26 Lim, D. H. et al. In vivo Large-Scale Cortical Mapping Using Channelrhodopsin-2 Stimulation in Transgenic Mice Reveals Asymmetric and Reciprocal Relationships between Cortical Areas. Front Neural Circuits 6, 11, doi:10.3389/fncir.2012.00011 (2012).

27 Fajardo, O., Zhu, P. \& Friedrich, R. W. Control of a specific motor program by a small brain area in zebrafish. Front Neural Circuits 7, 67, doi:10.3389/fncir.2013.00067 (2013).

28 Hira, R. et al. Spatiotemporal dynamics of functional clusters of neurons in the mouse motor cortex during a voluntary movement. J Neurosci 33, 1377-1390, doi:10.1523/JNEUROSCI.2550-12.2013 (2013).

29 Minlebaev, M., Ben-Ari, Y. \& Khazipov, R. Network mechanisms of spindle-burst oscillations in the neonatal rat barrel cortex in vivo. J Neurophysiol 97, 692-700, doi:10.1152/jn.00759.2006 (2007).

30 Kuhn, B., Denk, W. \& Bruno, R. M. In vivo two-photon voltage-sensitive dye imaging reveals top-down control of cortical layers 1 and 2 during wakefulness. Proceedings of the National Academy of Sciences 105, 7588, doi:10.1073/pnas.0802462105 (2008).

31 Spalletti, C. et al. Combining robotic training and inactivation of the healthy hemisphere restores pre-stroke motor patterns in mice. eLife 6, e28662, doi:10.7554/eLife.28662 (2017).

32 Chen, I. W., Papagiakoumou, E. \& Emiliani, V. Towards circuit optogenetics. Curr Opin Neurobio/ 50, 179-189, doi:10.1016/j.conb.2018.03.008 (2018).

33 Soor, N. S. et al. All-optical crosstalk-free manipulation and readout of Chronosexpressing neurons. J Phys D Appl Phys 52, 104002, doi:10.1088/1361-6463/aaf944 (2019).

34 Ronzitti, E., Emiliani, V. \& Papagiakoumou, E. Methods for Three-Dimensional AllOptical Manipulation of Neural Circuits. Front Cell Neurosci 12, 469, doi:10.3389/fncel.2018.00469 (2018).

35 Guo, Z. V. et al. Flow of cortical activity underlying a tactile decision in mice. Neuron 81, 179-194, doi:10.1016/j.neuron.2013.10.020 (2014).

36 Wang, X. et al. Deconstruction of Corticospinal Circuits for Goal-Directed Motor Skills. Cell 171, 440-455 e414, doi:10.1016/j.cell.2017.08.014 (2017).

37 Wise, S. P., Murray, E. A. \& Coulter, J. D. Somatotopic organization of corticospinal and corticotrigeminal neurons in the rat. Neuroscience 4, 8, doi:10.1016/03064522(79)90218-5 (1979).

38 Suter, B. A. \& Shepherd, G. M. Reciprocal interareal connections to corticospinal neurons in mouse M1 and S2. $J$ Neurosci 35, 2959-2974, doi:10.1523/JNEUROSCI.4287-14.2015 (2015). 
39 Card, N. S. \& Gharbawie, O. A. Principles of Intrinsic Motor Cortex Connectivity in Primates. J Neurosci 40, 4348-4362, doi:10.1523/JNEUROSCI.0003-20.2020 (2020).

40 Stark, E., Asher, I. \& Abeles, M. Encoding of reach and grasp by single neurons in premotor cortex is independent of recording site. J Neurophysiol 97, 3351-3364, doi:10.1152/jn.01328.2006 (2007).

41 Bansal, A. K., Truccolo, W., Vargas-Irwin, C. E. \& Donoghue, J. P. Decoding 3D reach and grasp from hybrid signals in motor and premotor cortices: spikes, multiunit activity, and local field potentials. J Neurophysiol 107, 1337-1355, doi:10.1152/jn.00781.2011 (2012).

42 Dombeck, D. A., Graziano, M. S. \& Tank, D. W. Functional clustering of neurons in motor cortex determined by cellular resolution imaging in awake behaving mice. $J$ Neurosci 29, 13751-13760, doi:10.1523/JNEUROSCI.2985-09.2009 (2009).

43 Riehle, A., Wirtssohn, S., Gruen, S. \& Brochier, T. Mapping the spatio-temporal structure of motor cortical LFP and spiking activities during reach-to-grasp movements. Frontiers in Neural Circuits 7, doi:10.3389/fncir.2013.00048 (2013).

44 Takahashi, K. et al. Large-scale spatiotemporal spike patterning consistent with wave propagation in motor cortex. Nat Commun 6, 7169, doi:10.1038/ncomms8169 (2015).

45 Balasubramanian, K. et al. Propagating Motor Cortical Dynamics Facilitate Movement Initiation. Neuron 106, 526-536 e524, doi:10.1016/j.neuron.2020.02.011 (2020).

46 Anderson, C. T., Sheets, P. L., Kiritani, T. \& Shepherd, G. M. Sublayer-specific microcircuits of corticospinal and corticostriatal neurons in motor cortex. Nat Neurosci 13, 739-744, doi:10.1038/nn.2538 (2010).

47 Hooks, B. M. et al. Laminar analysis of excitatory local circuits in vibrissal motor and sensory cortical areas. PLOS Biol 9, e1000572, doi:10.1371/journal.pbio.1000572 (2011).

48 Kelly, R. M. \& Strick, P. L. Cerebellar Loops with Motor Cortex and Prefrontal Cortex of a Nonhuman Primate. J. Neurosci 23, 8432-8444, doi:10.1523/JNEUROSCI.23-2308432.2003 (2003).

49 Grillner, S. \& El Manira, A. Current Principles of Motor Control, with Special Reference to Vertebrate Locomotion. Physiol Rev 100, 271-320, doi:10.1152/physrev.00015.2019 (2020).

50 Sauerbrei, B. A. et al. Cortical pattern generation during dexterous movement is inputdriven. Nature 577, 386-391, doi:10.1038/s41586-019-1869-9 (2020).

51 Sarasso, S. et al. Consciousness and Complexity during Unresponsiveness Induced by Propofol, Xenon, and Ketamine. Current Biology 25, 3099-3105, doi:10.1016/j.cub.2015.10.014 (2015).

52 Conti, E., Allegra Mascaro, A. L. \& Pavone, F. S. Large Scale Double-Path Illumination System with Split Field of View for the All-Optical Study of Inter-and Intra-Hemispheric Functional Connectivity on Mice. Methods Protoc 2, doi:10.3390/mps2010011 (2019).

53 Schindelin, J. et al. Fiji: an open-source platform for biological-image analysis. Nat Methods 9, 676-682, doi:10.1038/nmeth.2019 (2012).

54 Gulyas, M., Bencsik, N., Pusztai, S., Liliom, H. \& Schlett, K. AnimalTracker: An ImageJBased Tracking API to Create a Customized Behaviour Analyser Program. Neuroinformatics 14, 479-481, doi:10.1007/s12021-016-9303-z (2016).

55 Mardia, K. V. \& Jupp, P. E. Directional Statistics. Journal of applied Statistics 26, 949957, doi:10.1080/02664769921954 (1999). 
a
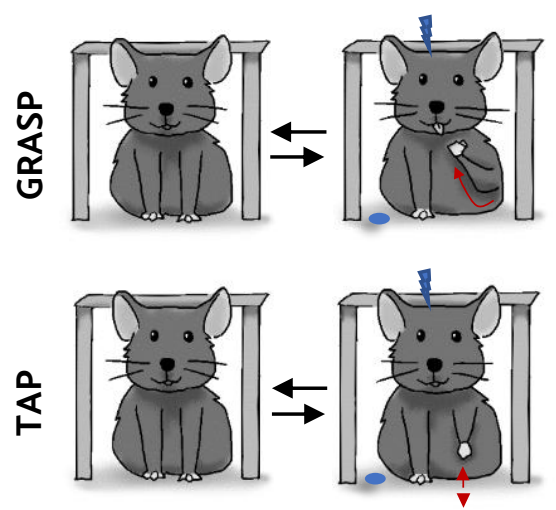

C

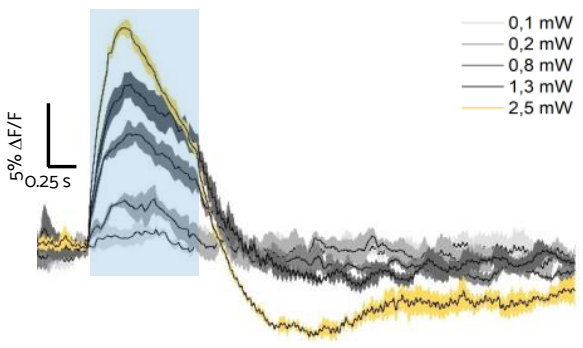

b
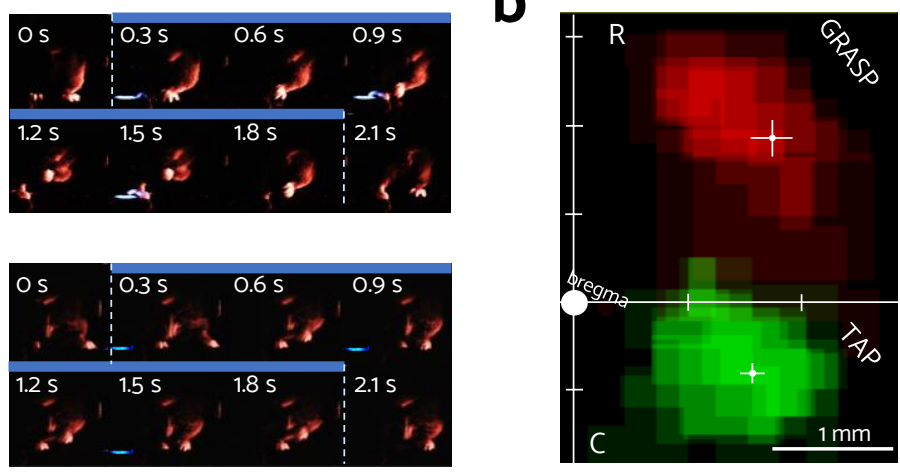

d

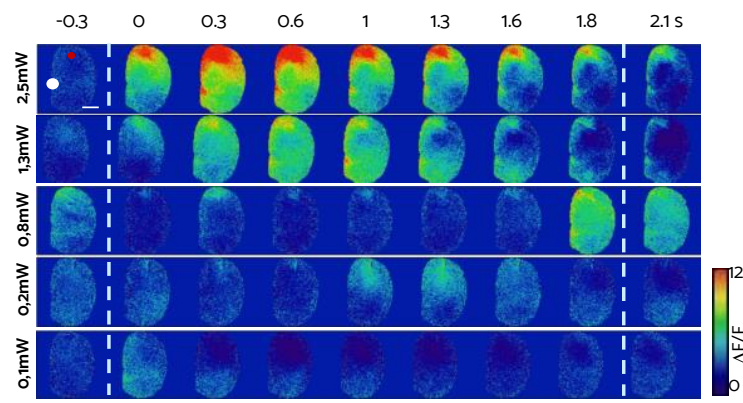

e
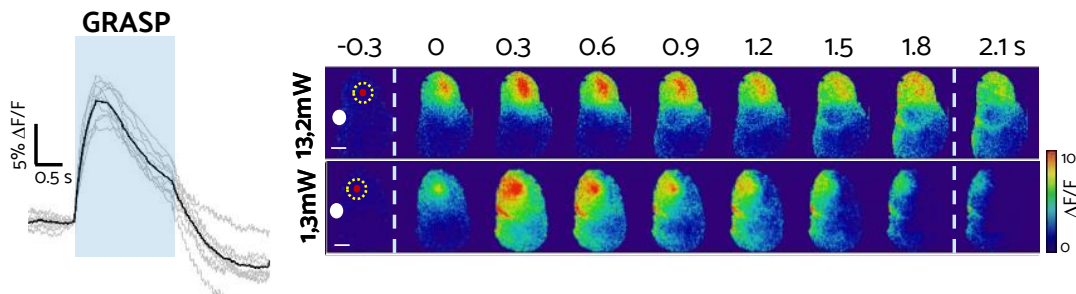

f
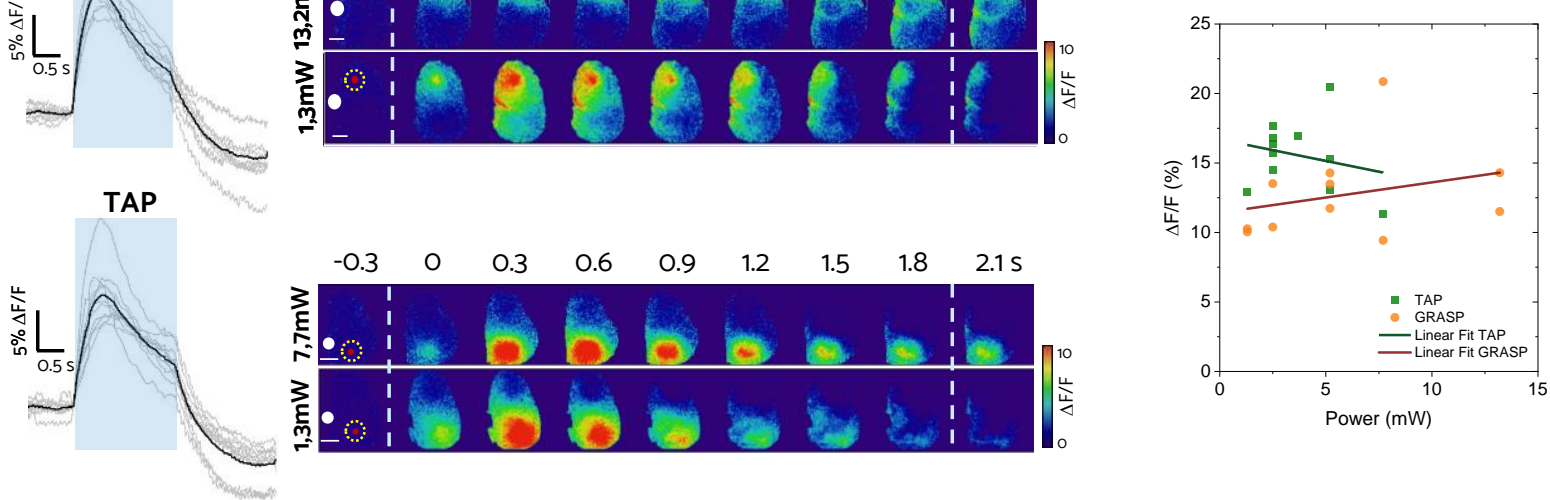

Fig. 2 Wide-field Imaging during light-based motor mapping reveals an activation threshold for movement execution

(a) Left, representative cartoons describing the evoked movements. Blue dots are the reflected laser stimuli representation. Red arrows indicate movement trajectories. Right, example frames from behavior recording during grasping-like movement (top) and locomotion-like movement (bottom). (b) Average light-based motor maps for GRASP movement (red) and TAP movement (green). White crosses represent the maps centers of mass and cross-bar lengths represent SEM (GRASP RC $=1,8 \pm 0,2$ $\mathrm{mm}$; GRASP LM $=1,8 \pm 0,2 \mathrm{~mm}$; TAP RC $=-1 \pm 0,2 \mathrm{~mm}$; TAP LM $=1,6 \pm 0,2 \mathrm{~mm} ; \mathrm{n}=$ 8). 
(c) Representative average calcium responses to the optogenetic stimulus train (10 ms, 16 $\mathrm{Hz}, 2 \mathrm{~s}$ ) at increasing laser powers. Yellow line represents the calcium response threshold associated with complex movement execution. Blue shadows represent the stimulation period. Shadows indicate SEM. (d) Representative wide-field image sequences of cortical activation at different laser powers. White dot indicates bregma. Red dot represents the site of stimulus. Dashed lines indicate the stimulus period. Scale bar $=1 \mathrm{~mm}$. (e) Left panel. Calcium transients evoked at the minimum laser power (TAP, $n=11$; GRASP, $n=11$ ). Black line indicates average calcium transient. Right panel. Representative image sequences of cortical activation at minimum evoking power in two extremes (lower and higher power. Red dot represents the site of stimulus. Dashed lines indicate the stimulus period. Yellow dashed dots indicate the $\mathrm{ROI}$ where the calcium transients were calculated. White dot indicates bregma. Scale bar $=1 \mathrm{~mm}$. (f) Linear regression between power thresholds and evoked calcium transient amplitudes $\left(\mathrm{TAP}_{\text {intercept }}=16.7 \pm 1.8 ; \mathrm{TAP}_{\text {slope }}=-0.3 \pm 0.4 ; \mathrm{GRASP}_{\text {intercept }}=11.4 \pm\right.$ 1.7; GRASP $\left._{\text {slope }}=0.2 \pm 0.2 ; \mathrm{n}=11\right)$. 

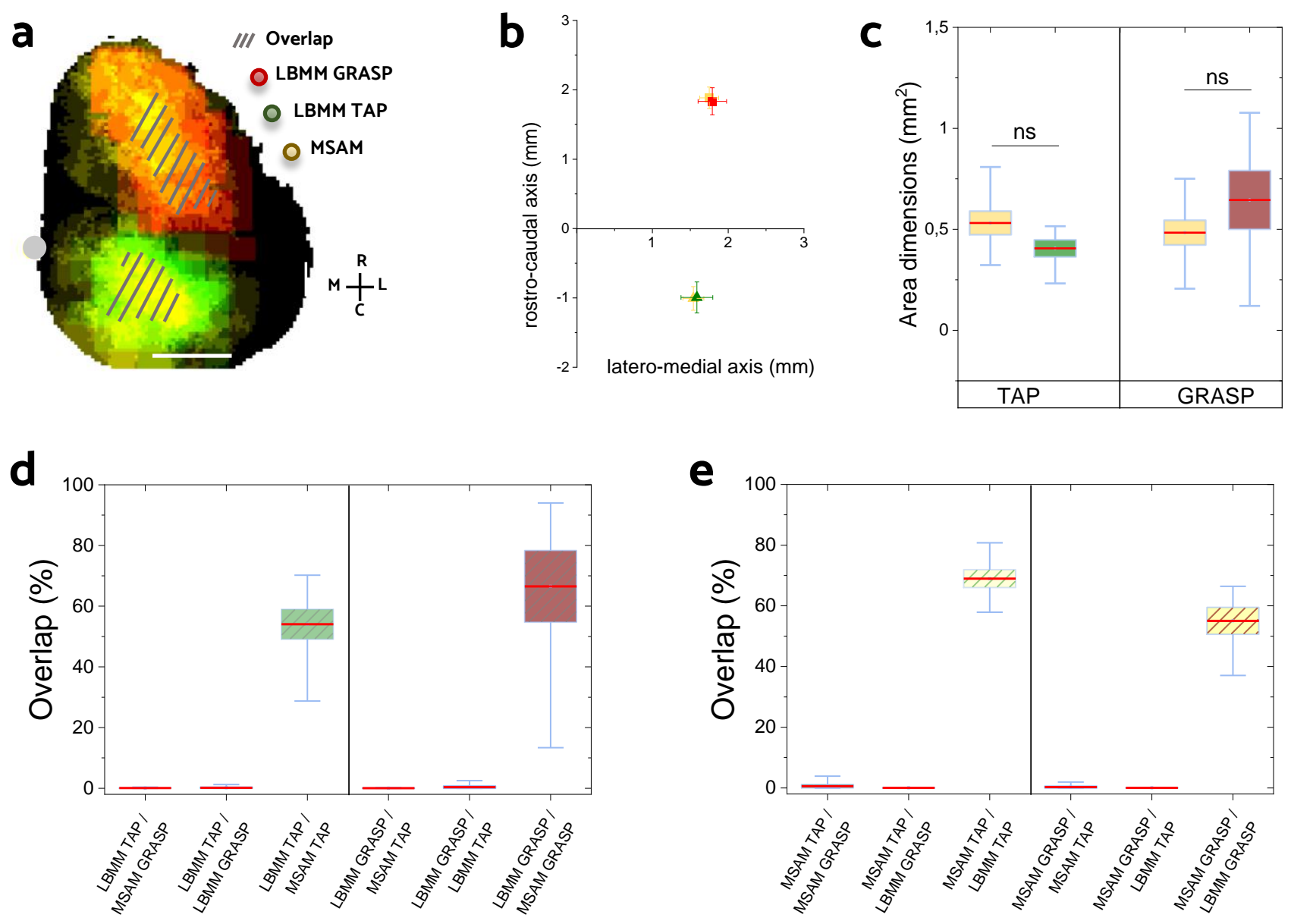

Fig. 3 Movement-specific cortical functional connectivity is bounded to discrete modules

(a) Representative scheme of cortical movement representations (LBMM: TAP $n=8$, red; GRASP $n=7$, green) and the related average movement-specific activation maps (MSAM yellow: TAP $n=8$; GRASP $n=7$ ). Gray dot indicates bregma. Scale bar $=1 \mathrm{~mm}(\mathbf{b})$ Centers of mass of the LBMM (TAP MSAM: LM $=1.5 \pm 0.1 \mathrm{~mm}$; RC $=-1.0 \pm 0.2 \mathrm{~mm}$; TAP LBMM LM $=1.6 \pm 0.2 \mathrm{~mm} ; \mathrm{RC}=-1.0 \pm 0.2 \mathrm{~mm} ; \mathrm{n}_{\mathrm{TAP}}=8$. GRASP $\mathrm{MSAM} L M=1.7 \pm 0.1 \mathrm{~mm} ; \mathrm{RC}=1.9$ $\pm 0.2 \mathrm{~mm}$; GRASP LBMM LM $=1.7 \pm 0.2 \mathrm{~mm}$; $R C=1.8 \pm 0.2 \mathrm{~mm}$; $\mathrm{n}_{\mathrm{GRASP}}=7$ ). Colors as in (a). Cross-bar lengths represent SEM. (c) Quantification of the area dimensions of the LBMMs and the relative MSAM $\left(\operatorname{TAP}_{\text {MSAM }}=0.53 \pm 0.06 \mathrm{~mm}^{2} ; \operatorname{TAP}_{\mathrm{LBMM}}=0.40 \pm 0.04 \mathrm{~mm}^{2}\right.$; $\mathrm{n}_{\text {TAP }}=8 ;$ GRASP $_{\text {MASM }}=0.48 \pm 0.06 \mathrm{~mm}^{2} ;$ GRASP $_{\text {LBMM }}=0.64 \pm 0.14 \mathrm{~mm}^{2} ; \mathrm{n}_{\text {GRASP }}=7$, two sample t-test). Comparison of the LBMMs (d) and the MSAMs (e) overlap ( $n=7$; see table $1)$. 


\section{a}

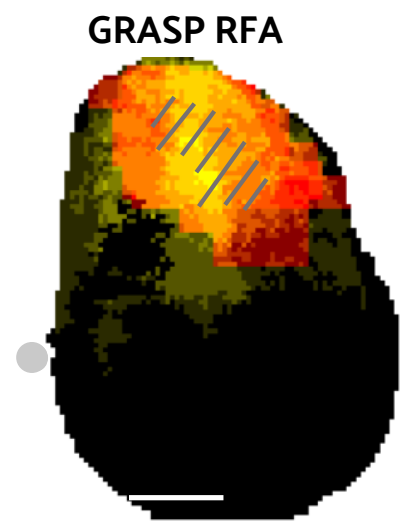

b

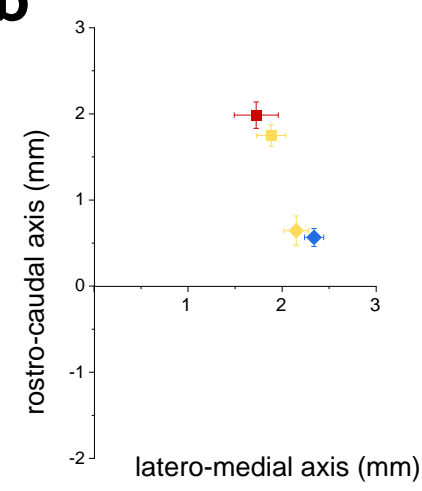

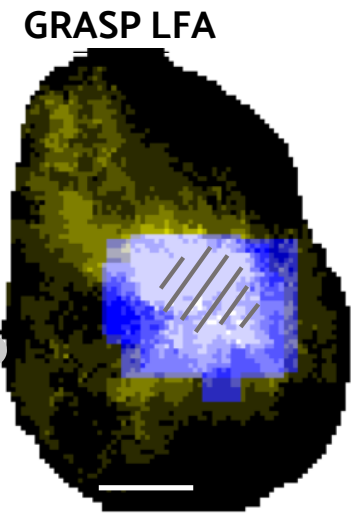

C

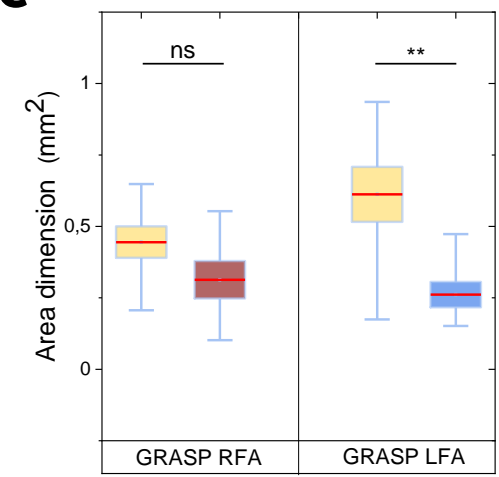

III Overlap

- LBMM GRASP RFA

LBMM GRASP LFA

- LBMMTAP

MSAM e

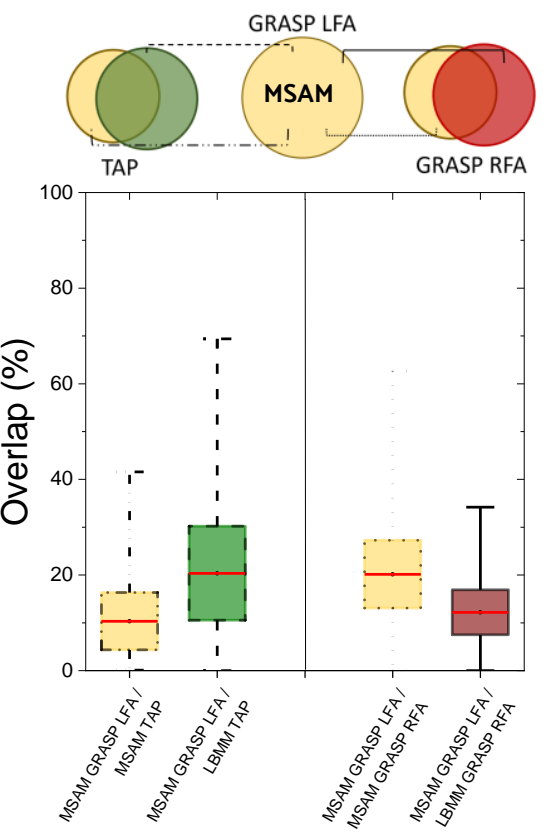

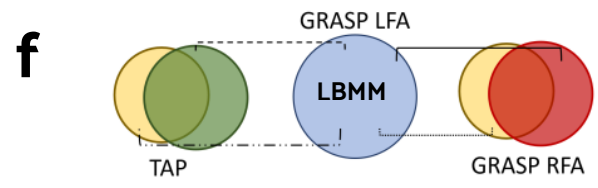

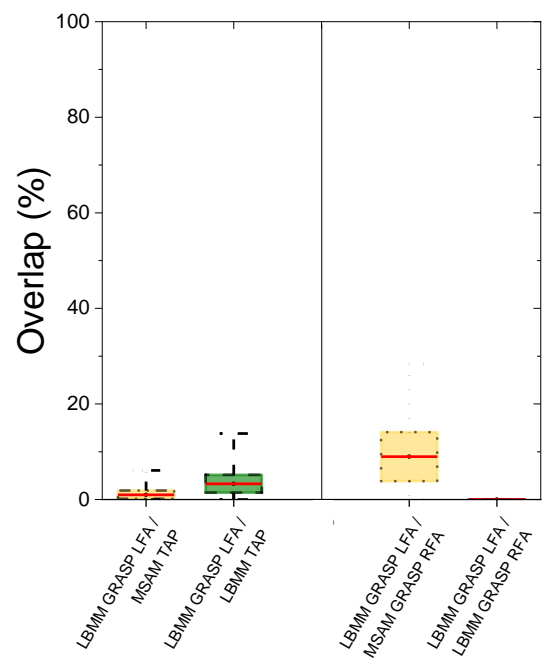

Fig. 4 Identification of the Lateral Forelimb Area (LFA) as a distinct grasping representation module

(a) Representative schemes of the cortical movement representations (GRASP RFA in red; GRASP LFA in blue) and their related average MSAM (yellow) $(n=7)$. Gray dot indicates bregma. Scale bar $=1 \mathrm{~mm}$. 
(b) Centers of mass of GRASP RFA LBMM (red), Grasp LFA LBMM (blue) and MSAMs (yellow) $\left(L B M M R F A_{R C}=2.0 \pm 0.2 \mathrm{~mm} ; R^{2} A_{L M}=1.7 \pm 0.2 \mathrm{~mm}\right.$ vs $L F A_{R C}=0.6 \pm 0.1 \mathrm{~mm}$; $\mathrm{LFA}_{\mathrm{LM}}=2.3 \pm 0.6 \mathrm{~mm} ; \mathrm{MSAM}_{\mathrm{RFA}} \mathrm{RC}=1.7 \pm 0.1 \mathrm{~mm} ; \mathrm{RFA}_{\mathrm{LM}}=1.9 \pm 0.2 \mathrm{~mm}$ vs $\mathrm{LFA}_{\mathrm{RC}}=$ $0.6 \pm 0.2 \mathrm{~mm} ; \mathrm{LFA}_{\mathrm{LM}}=2.1 \pm 0.1 \mathrm{~mm} ; \mathrm{n}=7$ ). (c) Quantification of the area dimensions of the LBMMs and the relative MSAM (GRASP RFA MSAM $=0.44 \pm 0.06 \mathrm{~mm}$; GRASP RFA LBMM $=0.31 \pm 0.07 \mathrm{~mm} 2$; GRASP LFA MSAM $=0.61 \pm 0.09 \mathrm{~mm} 2$; GRASP LFA LBMM $=0.26 \pm 0.04 \mathrm{~mm} 2 ; n=7,{ }^{*} p<0,01$ two sample t-test) . (d) Quantification of the overlay between MSAMs and LBMMs per movement category (GRASP RFA LBMM $/$ MSAM $=$ $61 \pm 3 \%$; GRASP LFA LBMM / MSAM $=77 \pm 2 \%$; GRASP RFA MSAM / LBMM $=50 \pm 12 \%$; GRASP LFA MSAM / LBMM $=38 \pm 6 \% ; n=7,{ }^{* * *} p<0,001$ two sample t-test). Red lines indicates means, boxes show the standard error range, whiskers length represents the extreme data points. (e) Multiple comparison between GRASP LFA MSAM and the other movement category maps ( $n=7$; see table 2). (f) Multiple comparison between GRASP LFA LBMM and the other movement category maps $(n=7$; see table 2$)$. 

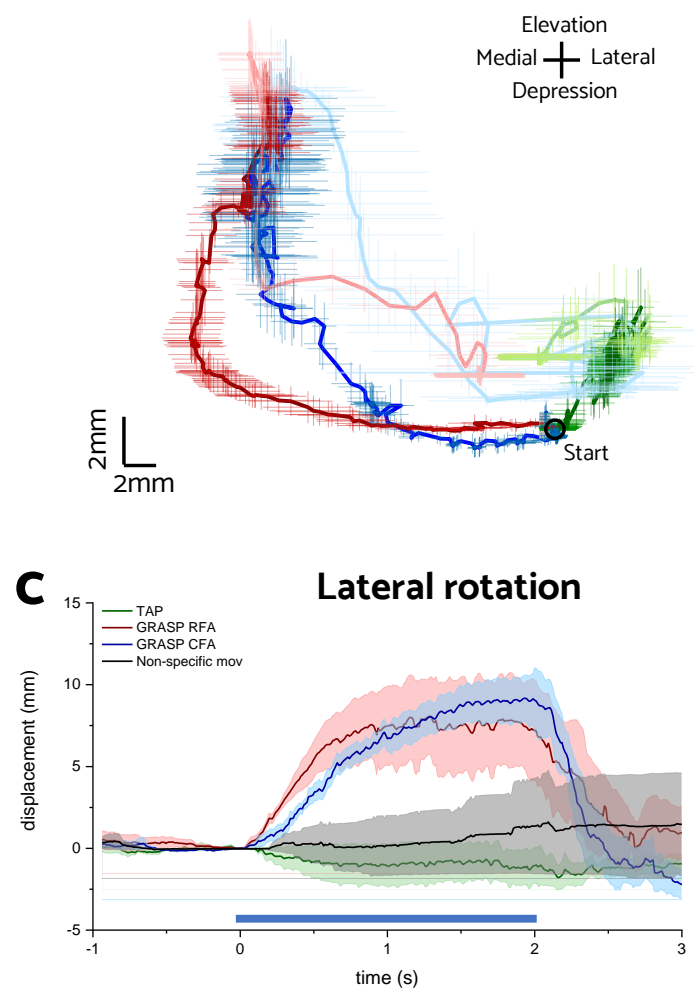

e

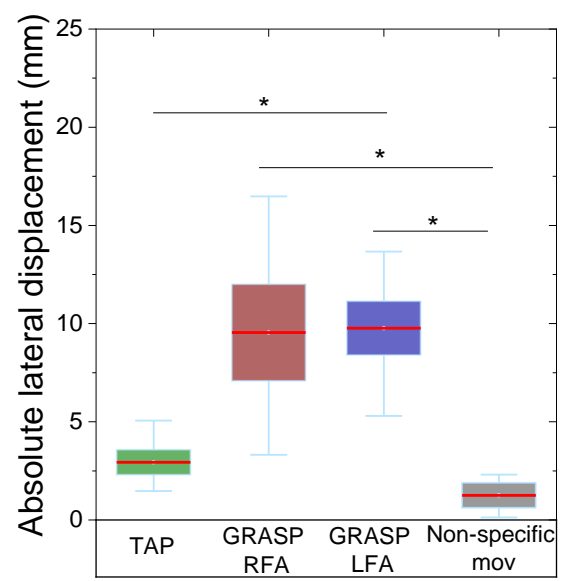

b

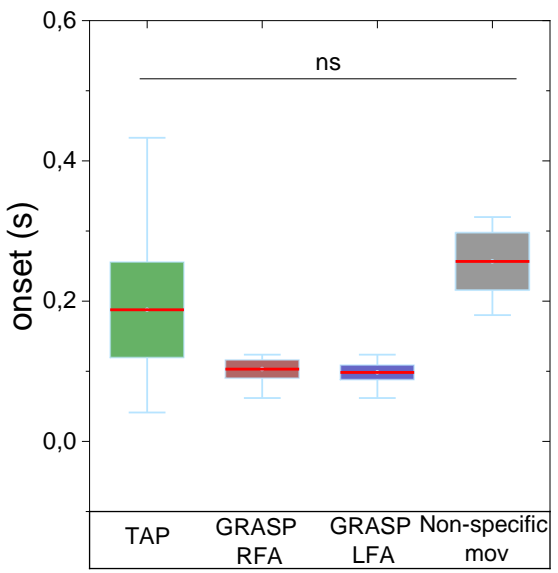

d

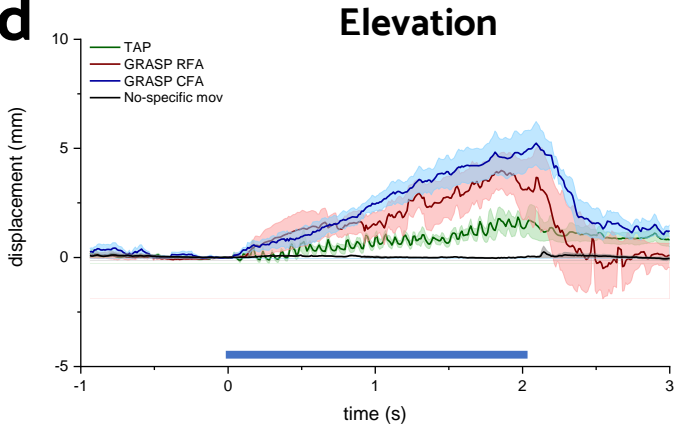

f

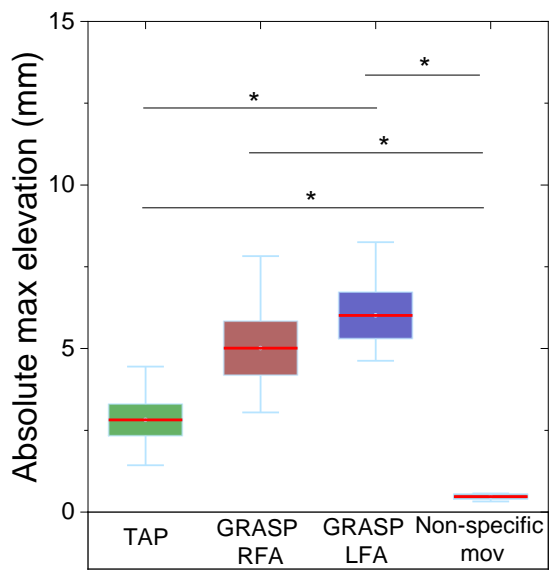

Fig. 5 Grasping-like behaviors evoked in RFA and LFA exhibit similar kinematic profiles

(a) Reconstruction of the mean trajectories evoked by optogenetic stimulation of GRASP RFA (red), GRASP LFA (blue), TAP (green) and non-specific movement (light gray). Dark traces show the movement trajectory during the $2 \mathrm{~s}$ stimulus period. Light traces show the movement trajectory $1 \mathrm{~s}$ post stimulation. Black circle indicates the forelimb start point. Error bars represent SEM. (b) Box and whisker plots showing the onset time per movement type (TAP, $0.19 \pm 0.07 \mathrm{~s}$; GRASP RFA, $0.10 \pm 0.01 \mathrm{~s}$; GRASP LFA, $0.10 \pm 0.01 \mathrm{~s} ; \mathrm{n}=5$; non-specific mov, $0.26 \pm 0.04 \mathrm{~s}, \mathrm{n}=3$; one-way ANOVA with post hoc Bonferroni test). (c) Medio-lateral forelimb displacement profiles. Dark traces represent the average medio-lateral displacement per movement category. Shadows indicate SEM. Blue line shows the stimulus period. (d) as in (c) mean elevation displacement along the $y$-axis. (e) Comparison of the absolute maximum displacement along the medio-lateral axis (TAP, $2.9 \pm 0.6$ $\mathrm{mm}$; GRASP RFA, $9.5 \pm 2.5 \mathrm{~mm}$; GRASP LFA, $9.8 \pm 1.4 \mathrm{~mm} ; \mathrm{n}=5$; non-specific mov, $1.2 \pm 0.6, \mathrm{n}=$ 3; $\mathrm{P}<0,05$ one-way ANOVA with post hoc Bonferroni test). (f) Comparison of the absolute maximum elevation (TAP, $2.81 \pm 0.48 \mathrm{~mm}$; GRASP RFA, $5.01 \pm 0.83 \mathrm{~mm}$; GRASP LFA, $6.01 \pm 0.71 \mathrm{~mm} ; \mathrm{n}=$ 5; non-specific mov, $0.47 \pm 0.08, n=3 ; p<0,05$ one-way ANOVA with post hoc Bonferroni test). 
a
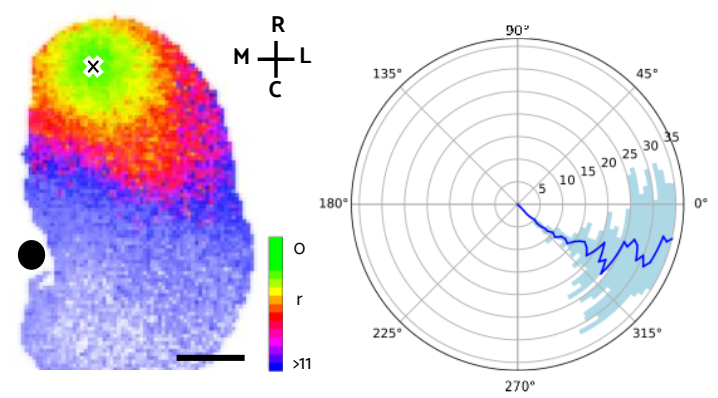

C

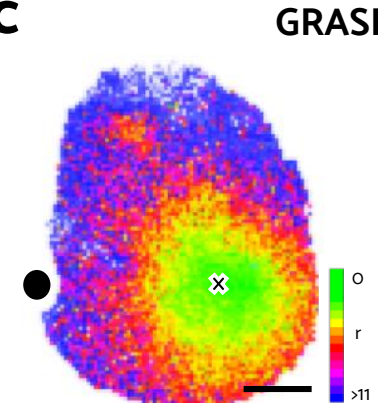

b

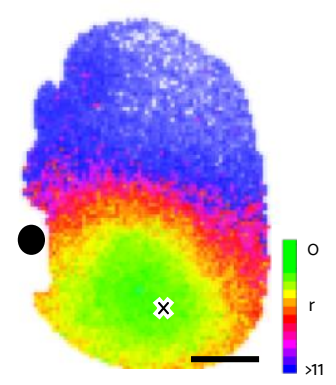

TAP

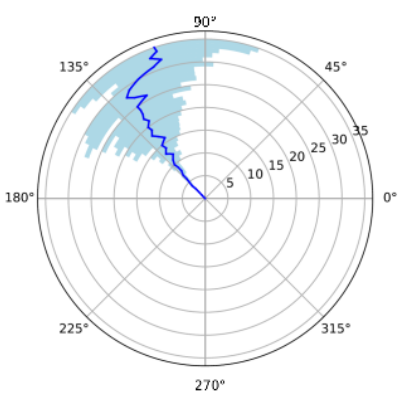

d

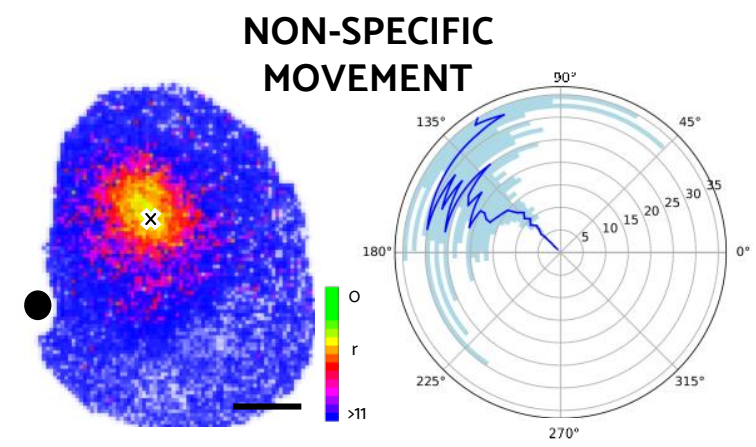

Fig. 6 Cortical activity propagation analysis reveals movement-specific spatiotemporal patterns of activation

(a) Left panel. Average map of the spatiotemporal activity propagation during GRASP RFA stimulation. Right panel. Polar plot, centered on the stimulation site, showing the average propagation direction. Blue line represents the radius-dependent circular mean. Shadow represents the standard deviation. As in (a) spatiotemporal activity propagation maps and polar plots are shown in (b), (c) and (d) for TAP, GRASP LFA and nonspecific movement stimulations respectively. Scale bars $=1 \mathrm{~mm}$. Color bar $=$ pixel ranks from 0 to $<11$. 
a
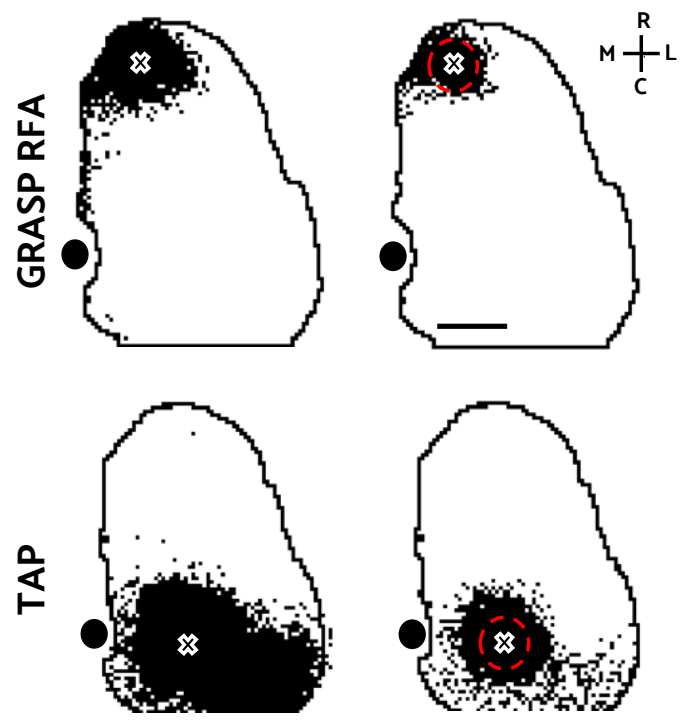

vehicle

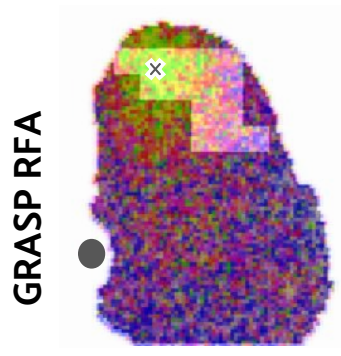

vehicle
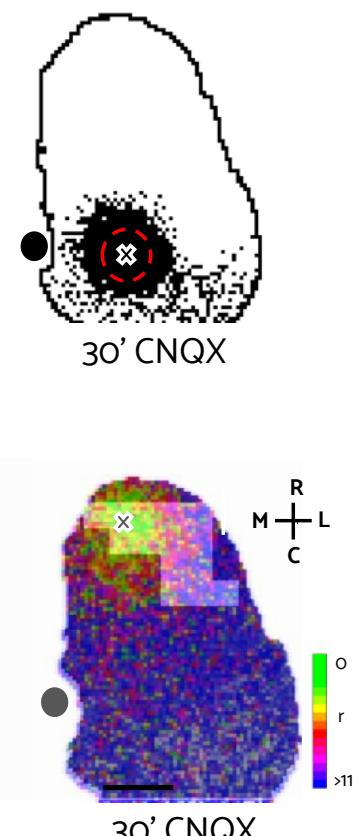

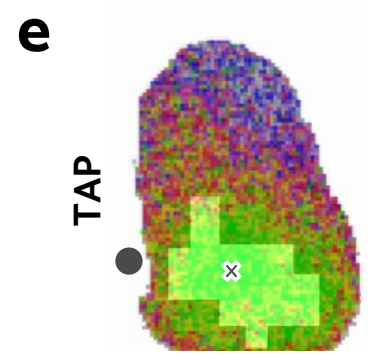

vehicle

g

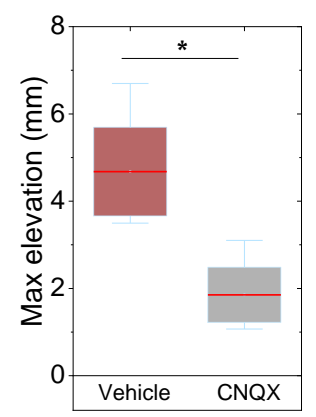

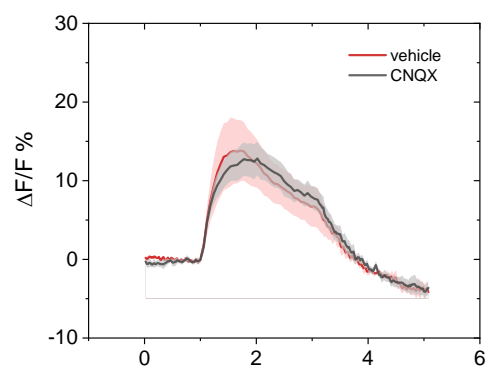

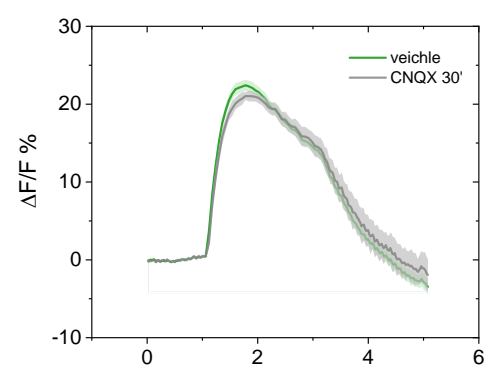

f

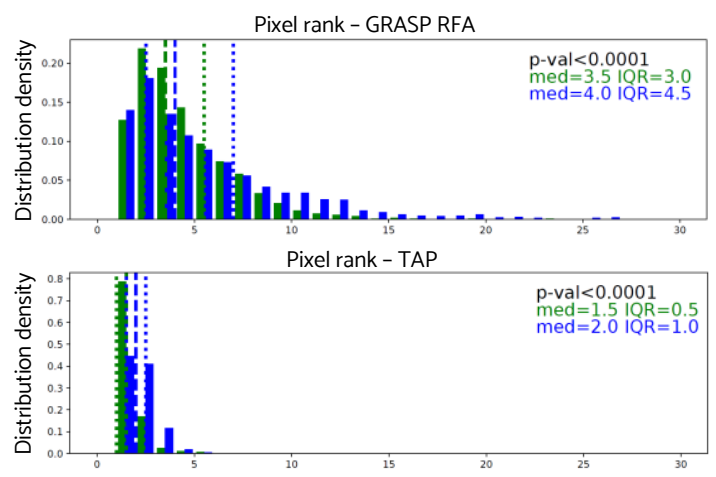

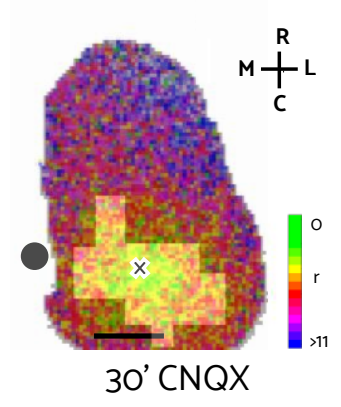

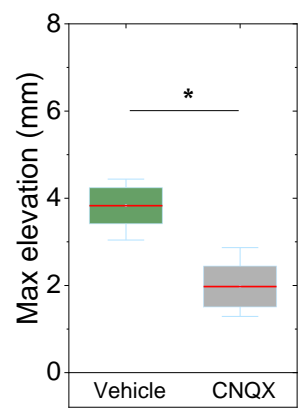

Fig. 7 Excitatory synaptic block leads to disruption of connectivity features associated with complex movement interference

(a) Representative MIP showing optogenetically evoked cortical activation during vehicle (left) and CNQX topical application (right) in RFA (top) and CFA (bottom). Cross represents the stimulus site. Red dashed lines indicates the CNQX topical application site. (b) Quantification of the effect of CNQX topical application on MSAM extension in RFA (top) and CFA (bottom) (GRASP: vehicle $=0.05 \pm 0.01 \mathrm{~mm} 2$ vs CNQX $=0.02 \pm 0.01 \mathrm{~mm} 2 ; \mathrm{n}=3$, * $p<0,05$, paired sample t-test; TAP: vehicle $=0.12 \pm 0.01 \mathrm{~mm} 2$ vs $C N Q X=0.093 \pm 0.026$ $\mathrm{mm} 2 ; \mathrm{n}=3,{ }^{*} \mathrm{p}<0,05$, paired sample t-test) 
(c) Averaged evoked calcium transient profiles in vehicle and following CNQX topical application in RFA (top) and CFA (bottom) (GRASP: vehicle $14.72 \pm 4.20 \Delta \mathrm{F} / \mathrm{F}$ vs CNQX 11.62 $\pm 3.66 \Delta \mathrm{F} / \mathrm{F} ; \mathrm{n}=3$, paired sample t-test; TAP: vehicle $25.80 \pm 1.31 \Delta \mathrm{F} / \mathrm{F}$ vs CNQX $21.26 \pm 0.63$ $\Delta \mathrm{F} / \mathrm{F} ; \mathrm{n}=3$, paired sample t-test). Shadows represent $\mathrm{SEM}$. Representative activity propagation maps of GRASP RFA (d) and TAP (e) showing the effect of CNQX topical application. Bright areas represent the LBMM. Scale bar $=1 \mathrm{~mm}$. Color bar $=$ pixel ranks from 0 to $<11$. (f) Pixel rank distribution of the region corresponding to the LBMM (bright) for GRASP RFA (top) and TAP (bottom), before (green) and after (blue) CNQX topical application $(n=3)$. Med $=$ median, $\mathrm{IQR}$ = interquartile range, Wilcoxon signed-rank test. (g) CNQX topical application effect on movement kinematics. Comparison of the absolute left-forelimb maximum elevation in vehicle (color) and CNQX topical application (gray) in RFA (left) and CFA (right) (GRASP: vehicle $4.6 \pm$ $1 \mathrm{~mm}$ vs CNQX $1.8 \pm 0.6 \mathrm{~mm} ; n=3$, * $p<0,05$, paired sample t-test; TAP: vehicle $3,8 \pm 0,4 \mathrm{~mm}$ vs CNQX $1.9 \pm 0.4 \mathrm{~mm} ; n=3,{ }^{*} p<0,05$, paired sample t-test). Red lines indicates means, boxes show the standard error range, whiskers length represents the extreme data points. 
a
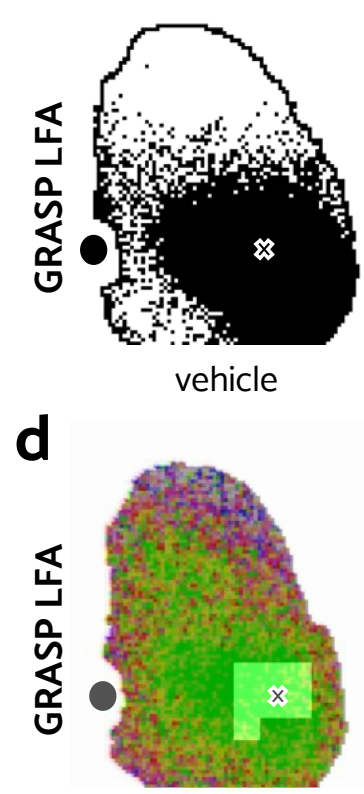

vehicle

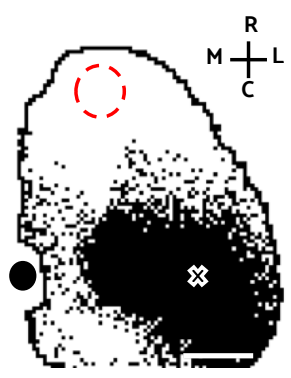

30 ' CNQX

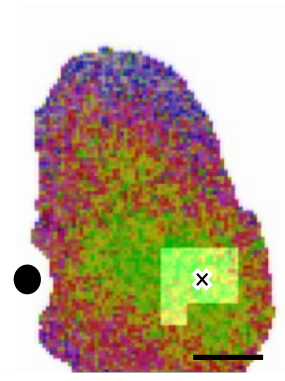

30' CNQX b

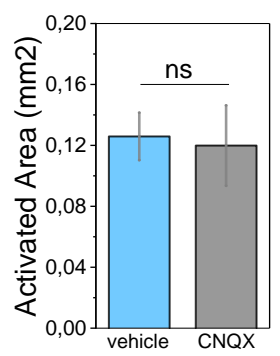

$\mathbf{e}$

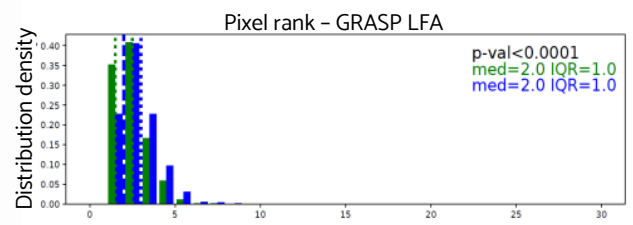

C

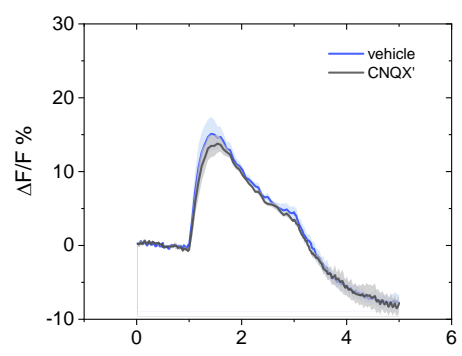

f

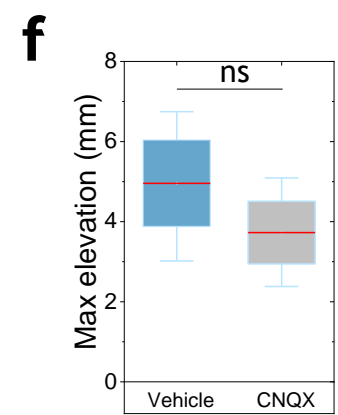

Fig. 8 LFA-evoked grasping does not require RFA activation

(a) Representative MIPs showing optogenetically evoked cortical activation in LFA during RFA topical application of vehicle (left) and CNQX (right). Cross represents the stimulus site. Red dashed line indicate the CNQX topical application site. (b) Quantification of the effect of CNQX topical application in RFA on LFA MSAM extension (vehicle $=0.12 \pm 0.01$ $\mathrm{mm} 2$ vs $C N Q X=0.11 \pm 0.02 \mathrm{~mm} 2 ; n=3$, paired sample t-test). (c) Averaged LFA evoked calcium transients profiles in vehicle and following CNQX topical application in RFA (vehicle $15 \pm 2 \Delta \mathrm{F} / \mathrm{F}$ vs $C N Q X 13 \pm 1 \Delta \mathrm{F} / \mathrm{F} ; \mathrm{n}=3$, paired sample t-test). Shadows represent SEM. (d) Representative activity propagation maps of GRASP LFA showing the effect of CNQX topical application in RFA. (e) Pixel rank distribution of the region corresponding to the LBMM (bright) for GRASP LFA, before (vehicle) and after CNQX topical application ( $\mathrm{n}=$ 3). Med = median, IQR = interquartile range, Wilcoxon signed-rank test. (f) Effect of RFA CNQX topical application on movement kinematics. Comparison of the absolute leftforelimb maximum elevation following LFA stimulation in vehicle and RFA CNQX topical application (vehicle $4.9 \pm 1,8 \mathrm{~mm}$ vs $\mathrm{CNQX} 3.7 \pm 1.4 \mathrm{~mm} ; \mathrm{n}=3$, paired sample t-test). Red lines indicate means, boxes show the standard error range, whiskers length represents the extreme data points. 
a

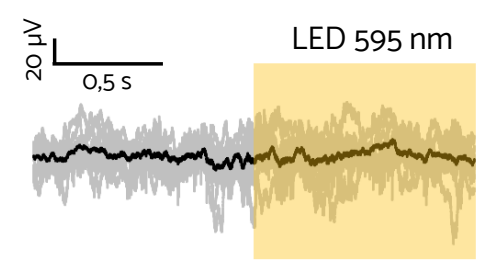

b

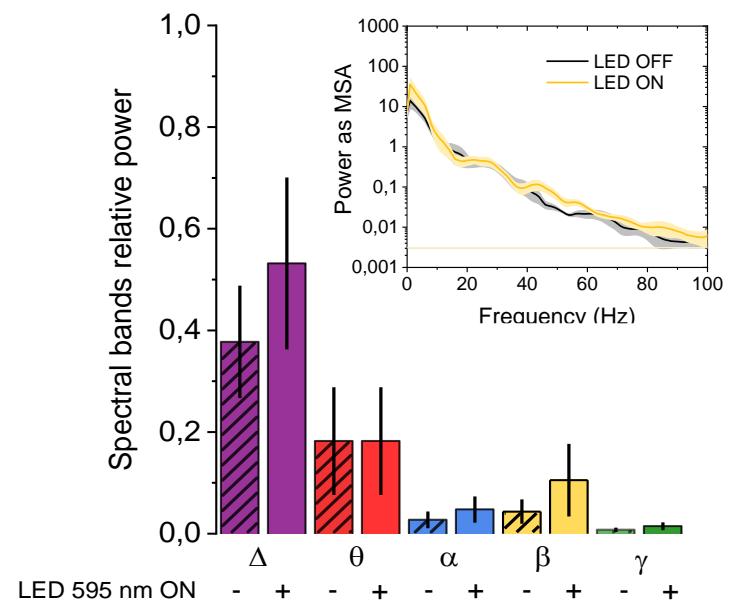

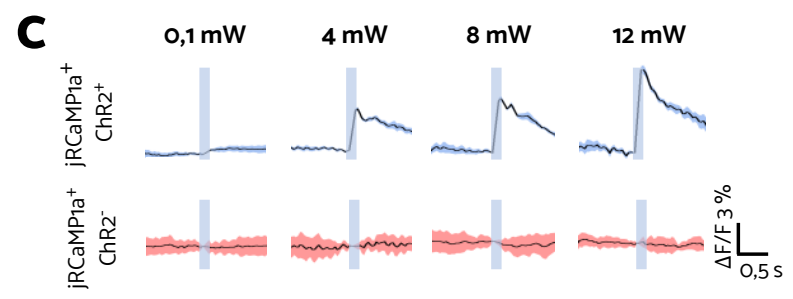

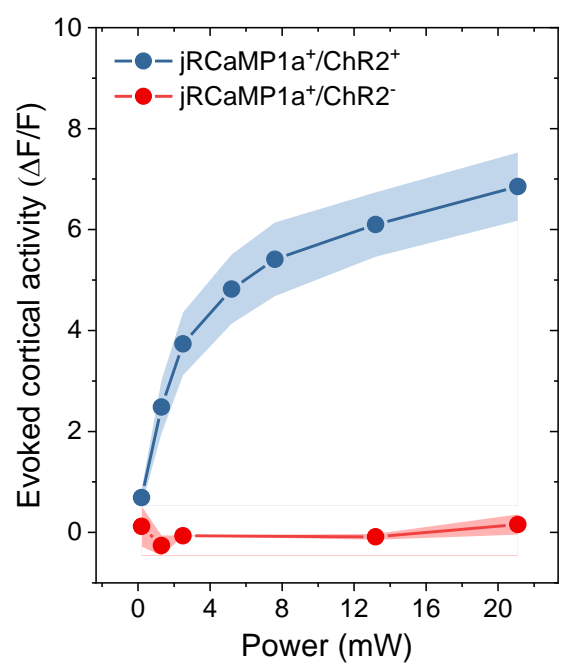

Supplementary fig. 1 Wide-field imaging of jRCaMP1a does not induce ChR2 crossactivation

(a) Representative traces of the LFP signal (gray; $n=10$ ) during $2 \mathrm{~s}$ of stimulus. Black line represents the mean signal. (b) Quantification of the spectral band relative power during 2 $s$ of peri-stimulus period ( $n=4$ mice; 10 stimuli per mice). Columns represent the averaged relative power for LFP frequency bands $(\boldsymbol{\Delta} ; \boldsymbol{\theta} ; \boldsymbol{\alpha} ; \boldsymbol{\beta} ; \boldsymbol{\gamma})$ during the dark period $(-2$ - $0 \mathrm{~s}$; patterned) and the LED illumination period $\left(0-+2 \mathrm{~s}\right.$; monochrome), $\mathrm{n}_{\text {mice }}=4, \mathrm{n}_{\text {stimuli }}$ $=10$, two-way ANOVA with post-hoc Bonferroni test, Data are presented as mean \pm SEM). Inset, representative averaged LFP power spectrum of 10 stimuli in dark period (grey) and LED illumination (Yellow). Shadows represents SEM. (c) Top panel. Representative traces showing the average calcium response (black) and the SEM (shadows) at increasing laser power $(0,1-10 \mathrm{~mW} ; 20 \mathrm{~ms}$ laser illumination) in mice expressing jRCaMP1a + ChR2 (blue) and only jRCaMP1a (red). Bottom panel. Correlation between evoked calcium activity and single pulse laser power in mice expressing jRCaMP1a+/ChR2+ (blue; $n=8$ ) and jRCaMP1a+/ChR2- (red; $n=2)$. Shadows represents SEM. 
a

movement
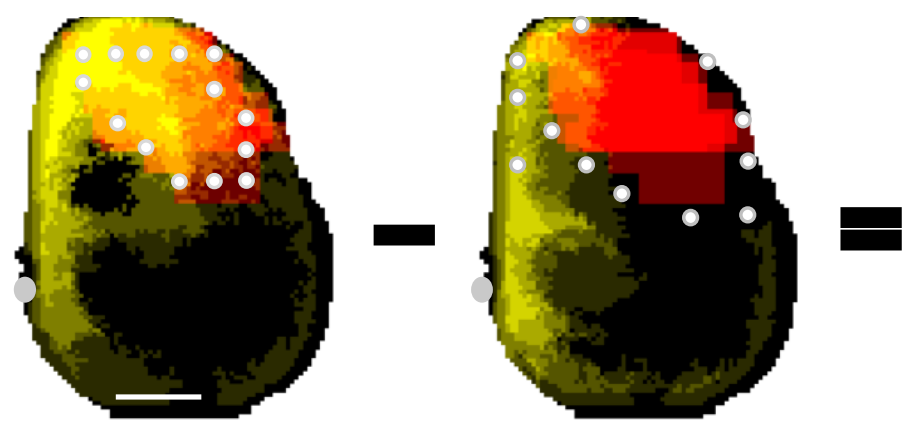

no-movement

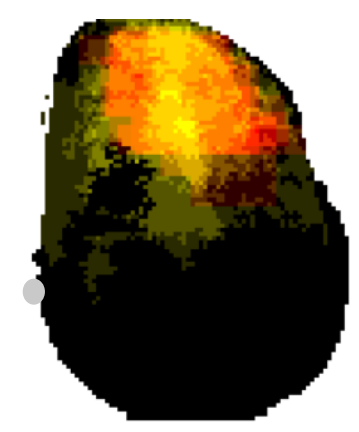

RFA
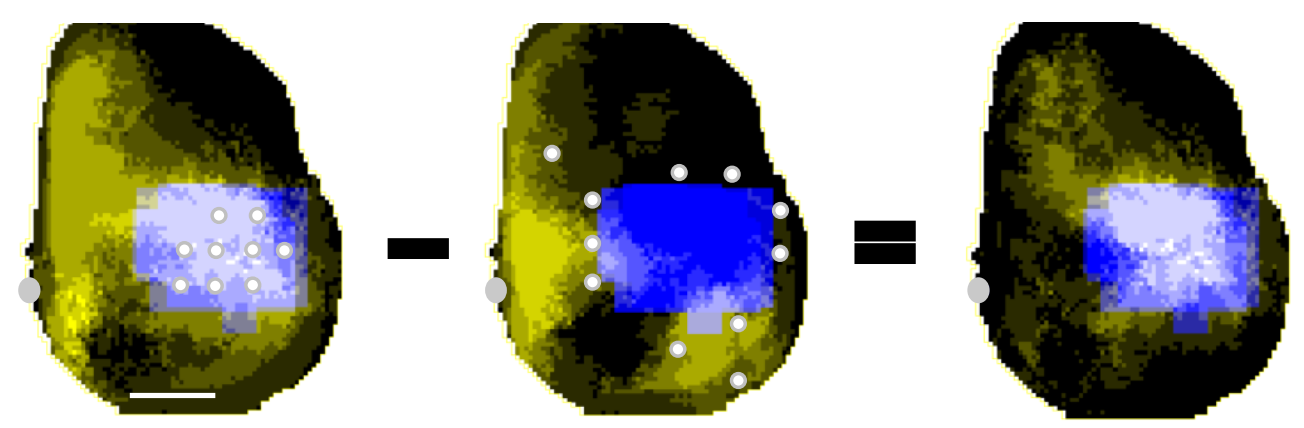

LFA

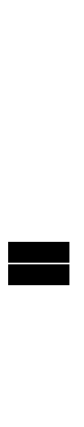

CFA

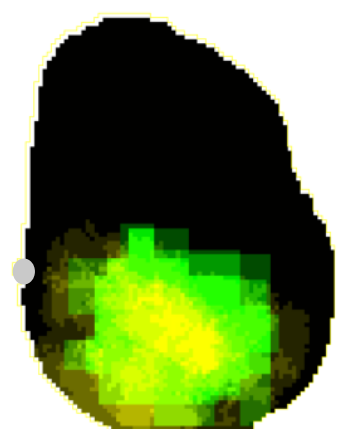

Supplementary fig. 2 Movement-specific activation maps (MSAMs) processing Average activation maps (yellow) obtained stimulating points (white dots) within the LBMM (colored areas) (a) and outside the LBMM (b). (c) Representative movementspecific activation maps (MSAMs) obtained by subtracting maps obtained in (b) to those obtained in (a). 
a

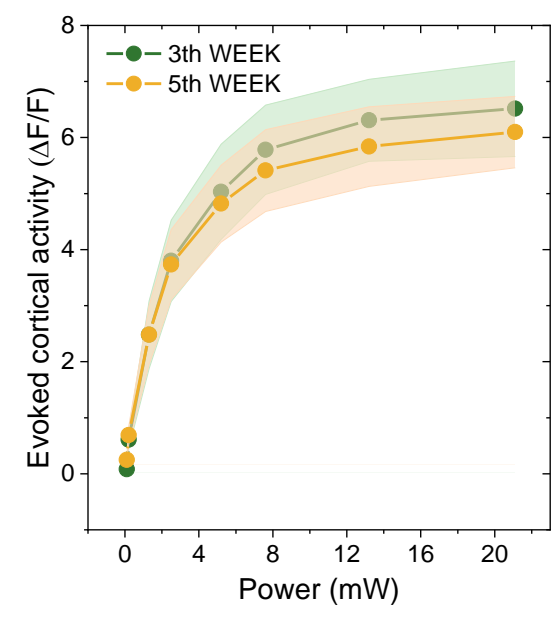

b

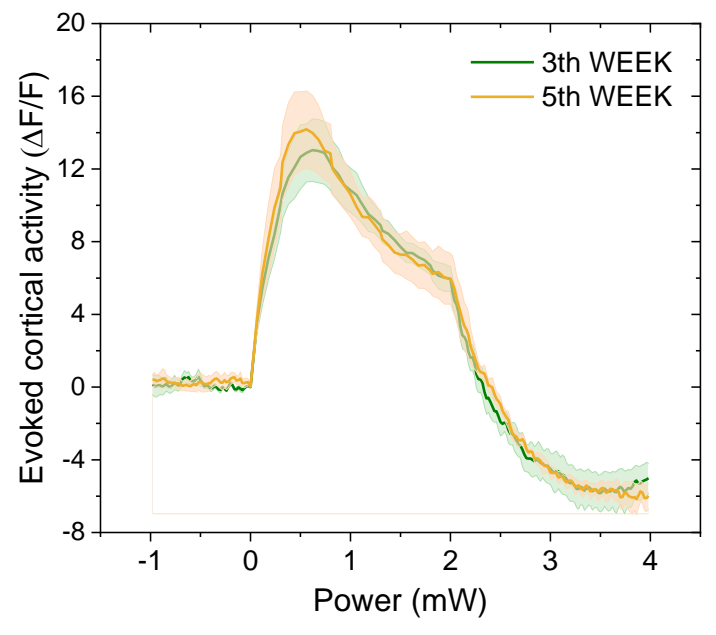

C

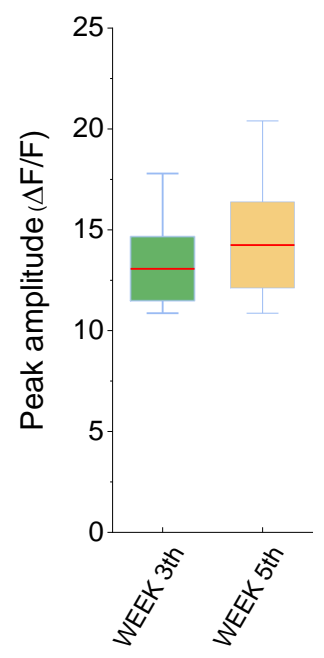

Supplementary fig. 3 Optogenetically-evoked calcium transients show long-term stability (a) Correlation between single laser pulse $(10 \mathrm{~ms})$ intensities and the calcium transient responses 3 and 5 weeks post-infection. (b) Calcium transients evoked by optogenetic stimulus trains (20 ms, $16 \mathrm{~Hz}$; 2s) showing long-term profile stability. (c) Quantification of the stimulus train-evoked calcium transient amplitudes 


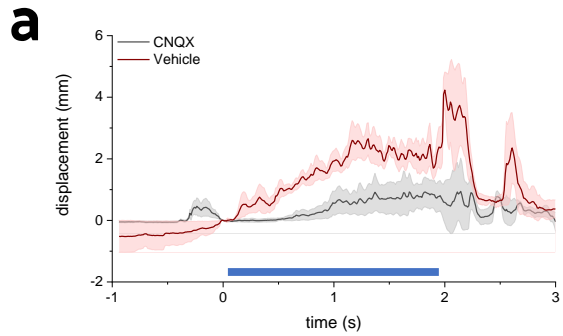

b

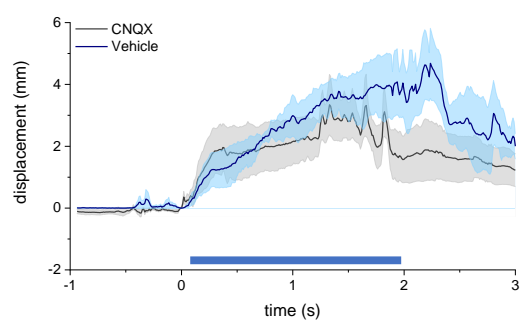

C

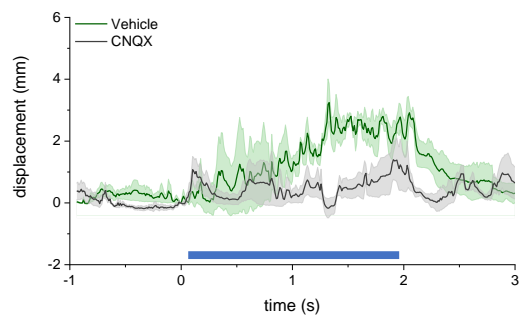

Supplementary fig. 4 Excitatoty synaptic transmission block disrupts the complex forelimb movement execution

(a) Forelimb displacement during GRASP RFA before and after CNQX topical application $(n=3)$. Blue line indicates stimulation period. Dark traces represent the average and shadows represent SEM. (b) and (c) show the same analysis for GRASP LFA and TAP respectively. 


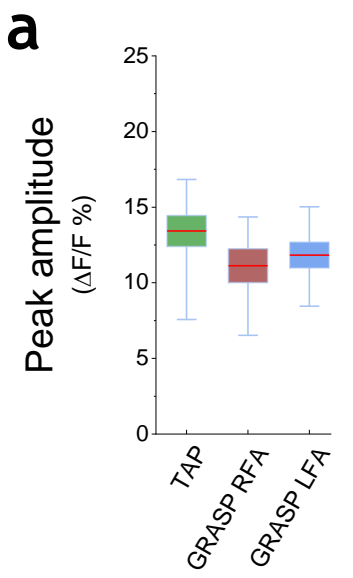

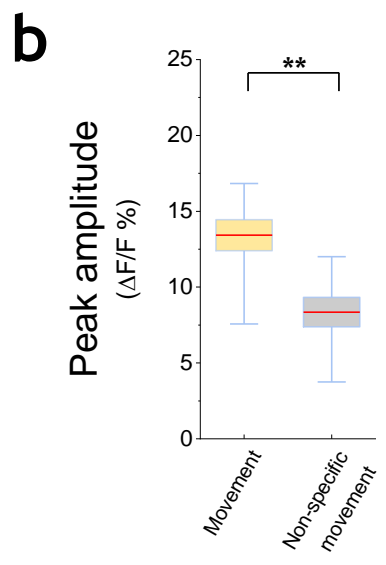

TAP

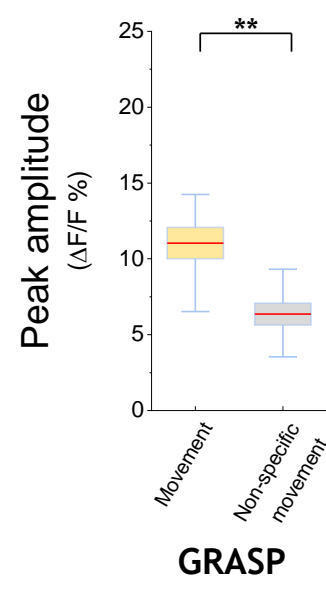

RFA

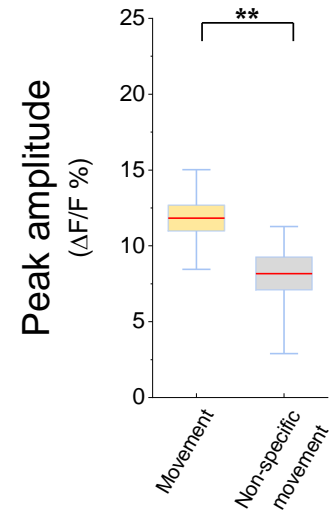

GRASP

Supplementary fig. 5 Comparison of average calcium transient properties between movement categories

(a) Comparison of evoked calcium transient amplitudes between movement categories (TAP 13.4 \pm 1.0 ; GRASP RFA $11.1 \pm 1.1 \Delta \mathrm{F} / \mathrm{F} ;$ GRASP LFA $11.8 \pm 0.8 \Delta \mathrm{F} / \mathrm{F} ; \mathrm{n}=7$, oneway ANOVA with post hoc Bonferroni test) (b) Comparison of the calcium transient amplitudes obtained stimulating within the LBMM (Movement) and outside the LBMM (non-specific movement) per movement classes (TAP: Movement $=13.4 \pm 1.0 \Delta \mathrm{F} / \mathrm{F}$ vs Non-specific movement $8.3 \pm 0.9 \Delta \mathrm{F} / \mathrm{F} ;$ GRASP RFA: Movement $=11.1 \pm 1.0 \Delta \mathrm{F} / \mathrm{F}$ vs Non-specific movement $6.3 \pm 0.7 \Delta \mathrm{F} / \mathrm{F} ;$ GRASP LFA: Movement $=11.8 \pm 0.8 \Delta \mathrm{F} / \mathrm{F}$ vs Non-specific movement $8.1 \pm 1.0 \Delta F / F ; n=7,{ }^{* *} p<0,01$ two sample t-test). 